For(e)Dialogue • Vol 4: Special Issue 1: Media and the Far-Right

\title{
Assessing the influence and reach of digital activity amongst far-right actors: $A$ comparative evaluation of mainstream and 'free speech' social media platforms
}

Hillary Woodworth McNerney ${ }^{1}$, Billy Spann², Esther L. Mead ${ }^{2}$, Joseph Kready ${ }^{2}$, Thomas Marcoux ${ }^{2}$, Nitin Agarwal ${ }^{2}$

${ }^{1}$ Regent Universiry, ${ }^{2}$ University of Arkansas at Little Rock

Published on: Jan 18, 2022

License: Creative Commons Attribution 4.0 International License (CC-BY 4.0). 
Hillary Woodworth McNerney, Regent University, hillmcn@mail.regent.edu

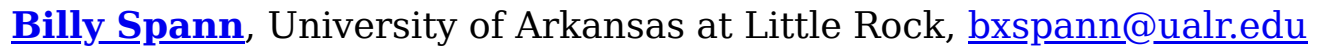

Esther L. Mead, University of Arkansas at Little Rock, elmead@ualr.edu

Joseph Kready, University of Arkansas at Little Rock, jkready@ualr.edu

Thomas Marcoux, University of Arkansas at Little Rock, txmarcoux@ualr.edu

Nitin Agarwal, Univerisity of Arkansas at Little Rock, nxagarwal@ualr.edu

\section{Abstract}

Mainstream social media platforms including Twitter, Facebook and YouTube, became more rigorous at the end of 2020 in implementing content moderation measures in efforts to combat false information related to the Covid-19 virus and election security in the United States of America. Some users viewed these measures as hostile towards various ideologies, prompting them to adopt alternative platforms for viewing and disseminating content (Abril 2021; Daly and Fischer, 2021). In 2020, the US Department of Homeland Security identified white supremacist extremists (WSE) as "the most persistent and lethal threat in the homeland" (DHS, 2020, p. 17). WSE disseminate their messages to a broader public by stoking grievances about race, immigration, multiculturalism and police-related policy issues, while also coordinating with networks of similar groups to carry their messages further (DHS, 2020). Current research lacks an understanding of the role these alternative platforms play in shaping, disseminating and amplifying extremist messages. This study utilized sociocomputational methods to compare and contrast user behavior on the mainstream platforms, YouTube and Twitter, with the alternative social media platform Parler, during the two months before and after the 2021 January 6th U.S. Capitol attack. Toxicity assessment, topic stream analysis, social network analysis, and social cyber forensic analysis helped identify key far-right actors and illuminated cyber coordination and mobilization within these social networks. The findings reveal some surprising insights including that both toxicity and posting activity were far greater on mainstream platforms and that Parler displayed an extremely high rate of cross-media and cross-platform posting. 
Keywords: Alt-right, radicalization, social identity, connective action, topic modeling, toxicity, social network analysis, Parler, YouTube, Twitter

\section{Introduction}

In May 2019 the US Federal Bureau of Investigation (FBI) produced an intelligence bulletin that was the first of its kind, as it was solely focused on the threat from conspiracy theory-driven domestic extremists, including 'anti-government, identity based, and fringe political conspiracy theories' (FBI, 2019, p. 5). The report assessed those believers of these conspiracies were very likely to pose a threat to 'specific people, places, and organizations' as promoters of these conspiracies use the power of the internet to expand their influence network by 'crowdsourcing' their extremist ideology, 'fostering anti-government sentiment, promoting racial and religious prejudice, increasing political tensions, and occasionally driving groups and individuals to commit criminal or violent acts' (pp. 4-5). Similarly, in October 2020, the US Department of Homeland Security (DHS) released its annual Homeland Threat Assessment, which warned of the rise of domestic violent extremists (DVE), including in the form of white supremacist extremism (WSE), leading DHS to designate this group as 'the most persistent and lethal threat to the homeland' (p. 18).'

These government reports warn that extremists have moved online, where narratives and ideologies are amplified through social media channels that can reach individuals who are vulnerable to radicalization. Evidence of this phenomenon can be found in research that correlates the rise in alt-right activism with a sharp increase in hate crimes (Atkinson, 2018; Lyons, 2017; Wendling, 2018, as cited in Lux and Jordan, 2019 , p. 4). To define the term, 'alt-right,' Forscher and Kteily (2019) describe the psychology of group adherents as falling on a spectrum wherein on one end, the movement can be seen as primarily driven by anti-establishment and anti-globalist aims. And on the other end, the movement can be seen as possessing status anxiety and racial resentment as well as having the goal of advocating for group-based supremacy, particularly on behalf of whites (p. 92). There is also evidence that Americans are becoming increasingly comfortable with the idea that violence may be necessary to achieve their political goals (Forscher and Kteily, 2019, p. 90; Pape, 2021, p. 4). Mass mobilization, such as what occurred at the US Capitol on January 6, can be thought of as the final step in the radicalization process that first involves disseminating extremist messages to a vulnerable audience, which are then amplified by influential voices and channels to cultivate anger and motivation as well as to maintain compliance (Mirrlees, 2018; Stanton, 2021). Therefore, WSE and other 
extremist acts should no longer be viewed as stemming from lone wolves or isolated extremist groups, but rather reflect broader social movements that emerge online.

In this context, this study focuses on two research objectives. The first objective is to identify how the alt-right shape, disseminate, and amplify their radicalized messages to the broader public using social media platforms, including so-called 'free speech' or alternative platforms. And secondly, we aim to assess the reach, influence, and coordination amongst far-right and extremist groups operating on these platforms. We do this by leveraging socio-computational techniques to examine extremist behavior and identify tactics used by actors on these social networks.

The key findings of this study include:

- Identifying the tactics used by far-right users on social media platforms via amplifying content (deploying bots,) cross-platform coordination, influence tactics (such as follow back), and anonymization tactics (such as IP masquerading).

- Identifying groups of users that were coordinating mention activity to at least 10 common target users. These groups served as coordinated 'mention brokers' between the top users with the most influence and shortest network path.

- Toxicity analysis showed that there was higher toxic content (including hate speech, profanity, identity attack, insults, etc.) within YouTube comments discourse than Twitter and Parler. This is counterintuitive to what is typically believed about alternative platforms.

- Topic modeling revealed that the discourse on each platform has an identifiable general nature, but obvious shifts in topics occurred both leading up to and soon after the January 6, 2021 U.S. Capitol riot.

- Engagement analysis of YouTube channels (gain in subscribers and views) revealed that some YouTube channels may have had an amplified influence on viewers leading up to January 6 th.

The remainder of this paper is organized as follows: The literature review examines the relevant literature related to new media use by actors and social movements and the theories that help explain group-based activism. The methodology section highlights the data collection and processing methods as well as identifies the computational techniques used for analysis. The data analysis and results section compare and contrast the findings in relation to each platform analyzed. We then highlight the key findings. Lastly, we discuss the conclusions and offer suggestions for future work. 


\section{Literature Review}

Theorizing social movements within a single academic silo misses the nuances and complexities within them. Therefore, in this study we draw upon scholarship from the social science disciplines of communication and computational studies as well as information science to orient our findings around the political, linguistic, and psychological underpinnings of the alt-right movement and the new media environments they operate in.

\subsection{Social Media's Influence on Extremism}

'The Internet affords movements and activists the powers of mass communication' (Postmes and Brunsting, 2002, p. 294). The alternative media landscape was one of the early adopters of the internet, and subsequently of social media platforms, recognizing the potential to break free from mainstream gatekeeping. This allowed conspiratorial, anti-establishment, and fringe political groups to harness the autonomy of the internet for collective action-that is 'actions undertaken by individuals or groups for a collective purpose, such as the advancement of a particular ideology' (Postmes and Brunsting, 2002, p. 290). Further, literature on collective action demonstrates that the greater level of psychological attachment to a group an individual has, the more they are willing to participate in protests or social movements (Chan, 2010, p. 1317).

In extreme cases, alt-right adherents turn to acts of violence and terrorism to further their cause by utilizing 'technosocial' politics-shaped by social forces as well as the affordances of information and communication technologies-to create new avenues through which to rapidly diffuse their ideologies (Shahin and $\mathrm{Ng}, 2020$, p. 2419). Social media platforms struggle to keep extremist content meant to motivate violence from being posted due in part to the failure to identify the 'amalgamation of [linguistic] codes and abbreviations used that often only mean something to other far-right extremists' (Der Spiegel, 2019, n.p.). It is imperative, then, when researching social media platforms, for scholars to gain a full intertextual understanding of these digital field sites. For example, it is important to recognize that the hashtag symbol (\#) functions as a filing system, which can serve a clerical purpose that allows a topic to quickly be indexed and retrieved but also may serve a semiotic function that allows users to 'performatively frame what the comments are really about' (Bonilla and Rosa, 2015 , p. 5). Having this awareness requires researchers to consider the variations of meaning a hashtag may have for different users and groups. 


\subsection{User Migration to Alternative Platforms}

In recent years, several alternative social media platforms have arisen to address concerns about discrimination and censorship measures by mainstream platforms. For this study, we define alternative social media platforms as those that market themselves as an alternative to the perceived ideological bias of mainstream platforms, and which promise users freedom of expression by offering lax moderation measures. However, many content creators, including Conservative politicians who flocked to alternative platforms in recent years, have found it difficult to attract followers and are continuing to also use mainstream platforms. It is important to note that support by politicians for these alternative platforms plays a role in legitimizing the content found there, including disinformation, conspiracies, and divisive rhetoric (Walther and McCoy, 2021) which has the potential to incite certain individuals to adopt extremist views towards national politics (p. 101).

\subsection{Identity and Social Movements}

First and foremost, white supremacists, white nationalists, and members of the altright belong to an identity movement, one that is both political and mediated by racial relations. There are several theories that have been used to explain this orientation, including social identity theory, introduced by social psychologists Henri Tajfel and John Turner in the 1970s. In its simplest terms, social identity theory can be explained as a continuum of an individual's own views of themselves in association with others; both on an individual level (interpersonal) and as a member of a group (intergroup) (Tajfel and Turner, 1986). The extent to which a person lies nearer one extreme or the other on the continuum determines to what degree their behaviors align with those of other members within their group (Ibid). Those who show extreme forms of social identification with a group, are more likely to treat outgroup members with some degree of hostility. Further, studies on computer-mediated communication shows that collective identity is strengthened by the anonymity and immersion that occurs within online groups as demonstrated by a model known as Social Identity Model of Deindividuation Effects (SIDE) (Odag et al., 2019, p. 281; Postmes, Spears and Lea, 1998; Spears and Postmes, 2015). This amplification of social identity, as mediated by digital interactions, can both heighten polarization and facilitate deviant behavior (Douglas and McGarty, 2002, as cited in Odag, et al., 2019, p. 281). The SIDE model was further developed to explain group behavior in larger social movements. The Social Identity Model of Collective Action (SIMCA) highlights group identity in relation to the group's perceptions of social injustice towards the group and the 'perceived efficacy' of collective action (Odag et al., 2019, p. 282). A direct correlation can be 
made between the level of group identification and the willingness of group members to participate in social movements on behalf of the group (Postmes and Brunsting, 2002; Klandermans, 2002; Simon et al., 1998, as cited in Chan, 2010, p. 1317).

In addition, research finds that users who seek out more extreme and violent content, are also more likely to be affected by that content (Slater et al., 2003, as cited in Odag et al., 2019, p. 280). There is strong evidence of this 'reinforcing spiral mechanism' occurring on digital platforms. A study on the psycholinguistic patterns of online Reddit community 'r/altright' found evidence of increasing 'warning behaviors' during the six months before the subreddit was banned for violations (Grover and Mark, 2019, p. 202). The researchers found that 'fixation on race,' and 'a strong linear increase in hostile language' as well as a higher-than-average use of language indicating 'in-group associations' are all indicators that suggest an online group is becoming increasingly radicalized (p. 194). The researchers suggest this insight into social identity, collective action and radicalization could potentially predict future terrorist events (p. 203).

Drawing upon this understanding, our study identifies how these movements and activists who operate on the far-right ideological spectrum, mobilize 'connective action' on social media through utilizing perceptions of social, racial, ideological injustice towards themselves, and amplifying those narratives by deploying bots, using cross-platform coordination, and influence tactics to reach a wider audience. Our computational methods assessed the nature of each platform, identifying the individuals and groups most successful in spreading these narratives across social networks.

\section{Methodology}

In this section, we describe the methodological components used in this work to address the two objectives. First, we discuss our data collection and processing methods. We then provide a brief explanation of the models and analysis techniques used. These include toxicity assessment, topic streams, and social network analysis. Identifying dominant topics allows us to meet our objective of identifying how alt-right users shape their messaging. Toxicity analysis allows us to determine whether alt-right users leverage the contagion power of toxicity to disseminate and amplify their messaging. Social network analysis techniques assess the reach that these alt-right users have, the coordination methods that they use to extend that reach, and the overall influence that they have on the network. 


\subsection{Data Collection and Processing}

Based on a manual qualitative analysis of known alt-right public figures active on social media, a set of specific actors was identified and selected as seeds for preliminary data collection. These initial individuals were extrapolated from mentions made by social media users on the Reddit forum r/paleoconservative. This forum (known as a subreddit) often hosted discussions that align with extreme alt-right ideology such as denying the Holocaust, disproving of interracial marriage, opposing immigration, and indicating support for segregation. The subreddit was closed in September 2021 but we collected several screenshots in our archive going back as early as March 2021. One discussion on the subreddit included a list of YouTubers they believed were capable of rallying others to their cause amongst the broader YouTube audience. Users identified actors such as John Doyle, Steven Crowder, Ben Shapiro, Matt Walsh, Nick Fuentes, and many others as having this potential. And while many people would consider these influencers to be more in line with mainstream conservatism, the Reddit users identified them specifically because of their deceptively congruent positions, with one user posting about Steven Crowder, 'Crowder has some cringe views but brings certain aspects to more mainstream YouTube attention.' Another user identified Fuentes and Doyle as 'the most influential' because they said they enjoyed 'highly analytical work with a personality.' Therefore, these seed social media accounts were the starting point used to grow our influencer network of altright actors.

From this preliminary analysis, we were able to create a list of hashtags used by these actors. This hashtag list was then used to conduct subsequent phases of data collection and filtration for both Parler and Twitter. The starting Parler dataset was obtained from the data sharing platform, Zenodo (Aliapoulios et al., 2021). We extracted data from this Parler dataset based on our hashtag list. Twitter data was collected using the Twitter Academic API (Twitter API for Academic Research Product Track, 2021). YouTube data for our set of key actors was collected using the YouTube Data API according to the methodology by Kready et al. (2020). Then, various postprocessing steps (data cleaning, sorting, and grouping) were taken. Next, we calculated toxicity scores, identified the dominant topics of discussion, and applied social network analysis to the datasets.

\subsection{Toxicity Assessment}

Toxicity analysis was applied to our datasets based on a tool called Detoxify (Detoxity: Toxic Comment Classification with Pytorch Lightning and Transformers, 2021). 
Detoxify uses a Convolutional Neural Network (CNN) that is trained with word vector inputs to determine whether text could be perceived as "toxic" to a discussion. The API returns a probability score between 0 and 1 , with higher values indicating a greater likelihood of the toxicity label being applied to a piece of text. Since toxicity scores are based on a probability score of 0 to 1 , toxicity scores of 0.5 or greater are indicative of a piece of text that is labeled as "toxic".

\subsection{Topic Streams}

For each dataset, topic streams were generated based on the LDA (Latent Dirichlet allocation) topic modeling methodology by (Marcoux et al., 2021). The method includes the use of an LDA model that identifies the top topics in terms of distribution within a text corpus. The topic streams component of the method shows the evolution of those topics over time. The topic modeling component within the topic streams methodology allows us to detect patterns in text by clustering groups of words or expressions that are similar or appear most often together. This information allows us to quickly deduce what a set of text is talking about.

\subsection{Social Network Analysis}

We performed social network analysis by creating communication networks within our datasets. These included user-mention, user-URL, and user-hashtag networks. Each of these networks were analyzed using the open-source network analysis and visualization software tool Gephi (Bastian and Heymann, 2009). After creating network graphs, we used the Louvain community detection method (Blondel, et al., 2008) to separate the networks into groups of users with the densest connections between them. This type of analysis allowed us to quickly identify the key actors, communities of interest, URLs that were disseminated, and to find sub-groups that shared similar ideologies.

\section{Data Analysis and Results}

In this section, we discuss our data analysis and results of the datasets explored in this work. First, we present the key findings of our toxicity analysis and identification of the topics that dominated the discourse. Then, we present the results of our social network analysis including various network graphs to illustrate the key users on each platform. Finally, social network analysis provides a discussion of the results based on our datasets for each platform. Table 1 provides a summary of the datasets explored in this work in their entirety; and Table 2 provides a summary of the restricted datasets that focus on the timeline two months prior and post January 6, 2021. 
Table 1. Data summary for datasets analyzed in this work.

\begin{tabular}{|l|l|l|}
\hline Dataset & Records & Date Range \\
\hline Parler Posts & 18,079 & $2018-12-09-2021-01-09$ \\
\hline Twitter Posts & 19,223 & $2010-02-12-2021-06-19$ \\
\hline YouTube Channels & 6 & $2006-06-02-2018-10-10$ \\
\hline YouTube Videos & 5,115 & $2009-01-05-2021-06-19$ \\
\hline YouTube Comments & $10,002,035$ & $2009-01-05-2021-06-20$ \\
\hline $\begin{array}{l}\text { YouTube } \\
\text { Related/Recommended Videos }\end{array}$ & $6,068,057$ & $2006-01-09-2021-06-19$ \\
\hline
\end{tabular}

Table 2. Summary statistics for datasets reduced down to two months prior and post the January 6, 2021, United States Capitol Riot (November 1, 2020, to March, 1, 2021).

\begin{tabular}{|l|l|l|}
\hline Dataset & Records & Date Range \\
\hline Twitter & 4,516 & $2020-11-01-2021-03-01$ \\
\hline Parler* & 3,361 & $2020-11-01-2021-01-09$ \\
\hline YouTube Comments & $1,664,123$ & $2020-11-01-2021-03-01$ \\
\hline YouTube Videos & 548 & $2020-11-01-2021-03-01$ \\
\hline
\end{tabular}

*Parler data, which was obtained from an outside source, is cut off, due to the platform being shut down by Amazon on January 10, 2021.

\subsection{Toxicity Analysis}

In this section, we discuss the results of our toxicity assessment of the datasets for each platform. The interjection of toxicity within social media discourse has been shown to have interesting effects on the flow of conversations as indicated by analyses of network graphs (Obadimu et al., 2021). These effects include the creation of echo chambers wherein toxic comments become amplified, as well as the creation of polarization among users. 


\section{Analysis of Toxicity for Entire Datasets}

First, we identified the toxicity statistics and trends for each platform over the original timeframe of our datasets. As can be seen from Figure 1 (left), the average toxicity on a yearly basis between 2018 and 2021 was relatively low on each platform.

Consistently, however, toxicity was highest within the YouTube comments discourse, and the lowest within the Parler discourse. Figure 1 (right) shows the detailed toxicity distribution characteristics using the box plot that displays minimum, maximum, median, first quartile, and third quartile.

Figure 1. Toxicity of Parler posts, Twitter posts, YouTube videos, and YouTube comments for complete datasets. Left image shows the yearly average toxicity for 2018-2021 and the right image shows the box plot of the toxicity distribution for each dataset for the entire period.

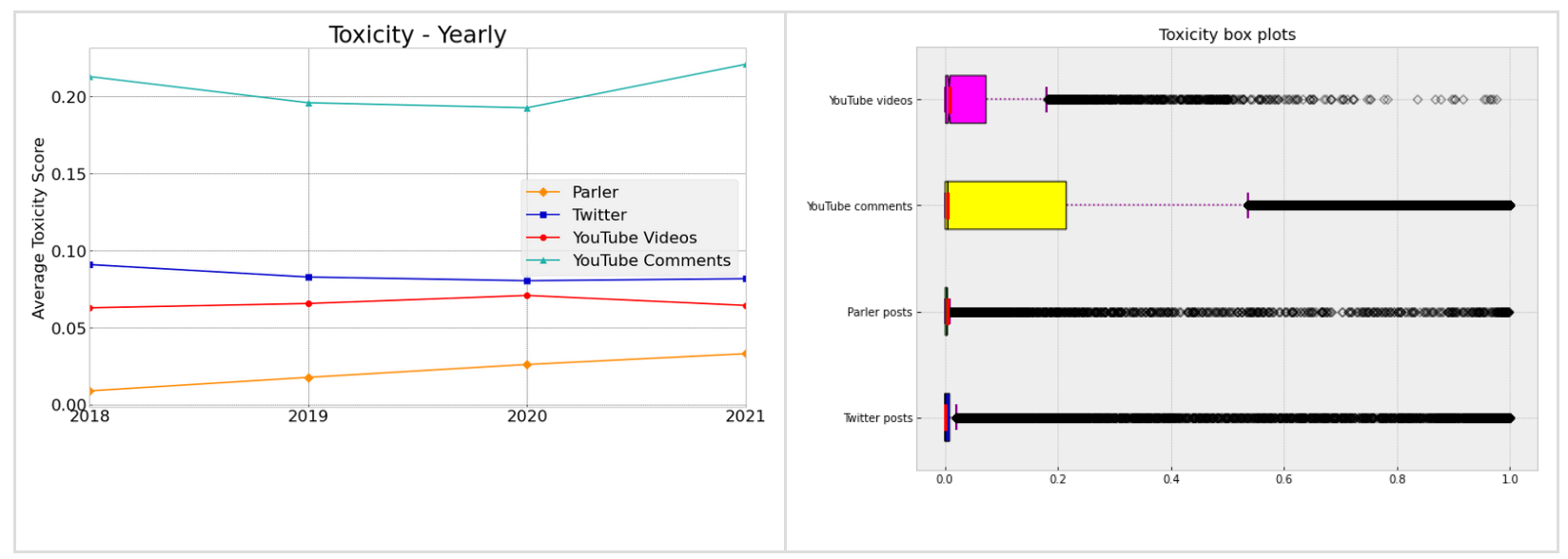

Next, we homed in on the toxicity statistics and trends for all platforms by reducing their respective datasets down to two months prior and post January 6, 2021. Figure 2 (left) reveals that the average toxicity level for each platform remained consistent but registered a jump in the weeks before and after January 6. Figure 2 (right) shows the detailed toxicity distribution characteristics using the box plot that displays minimum, maximum, median, first quartile, and third quartile.

Figure 2. Toxicity of Parler posts, Twitter posts, YouTube videos, and YouTube comments from November 1, 2020, to March 1, 2021. Left image shows the weekly average toxicity and the right image shows the box plot of the toxicity distribution for each dataset for the selected period.

Note: Parler data, which was obtained from an outside source, cuts off on January 8 , 2021. 


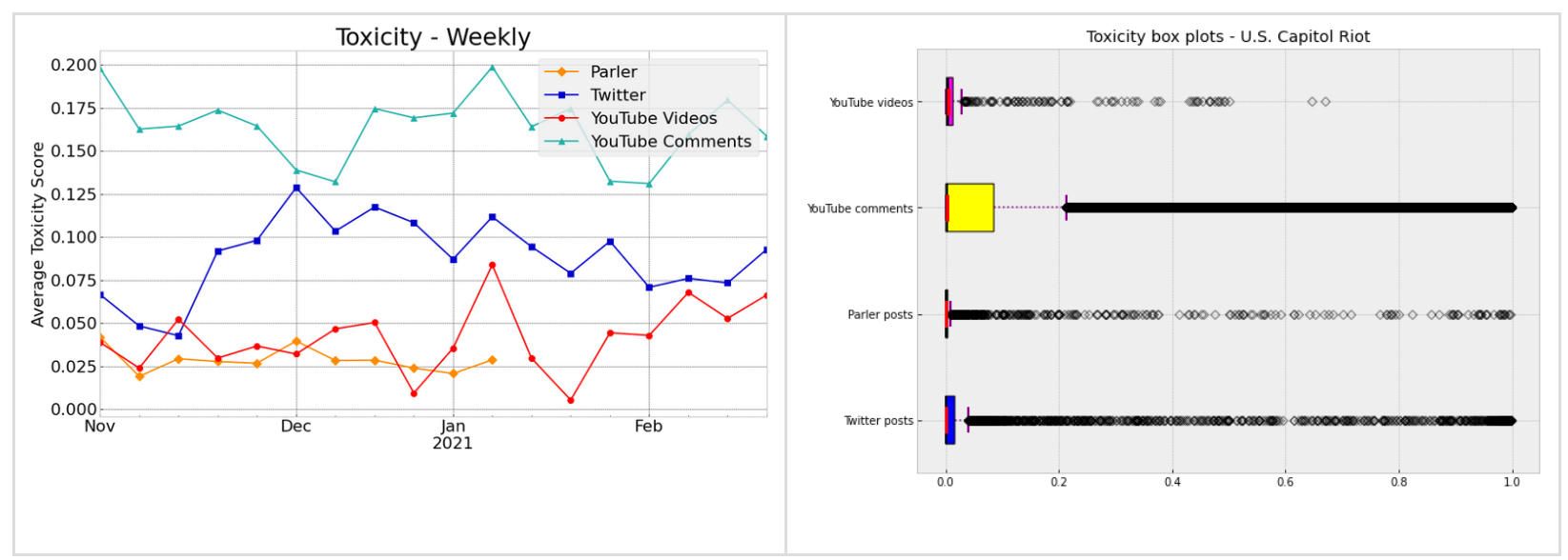

\subsection{Topic Streams Analysis}

In this section, we discuss the results of our topic streams analysis based on LDA topic modeling. First, we discuss the topics for the original timeframe of our analysis. This serves as a baseline for a comparison of our subsequent topic streams analysis of the two-month timeframe around the January 6, 2021, Capitol riot. This comparison allows us to determine whether there were any indications of divergent topics both leading up to and following this event.

\section{Analysis of Topic Streams for Entire Datasets}

Figure 3 reveals the dominant topics within the Parler discourse for the entire time frame of our original dataset. The most dominant topic streams combined discussions around Trump, his press secretary Kayleigh McEnany, and the far-right blog the Geller Report (Topic 13). These topics were dominant up until September of 2020, when the topics shifted from Trump and his supporters (Topic 0) to Trump and the Presidential election, campaign discussions, discourse about Joe Biden, and links shared from the far-right news website, the Gateway Pundit (Topic 15). This technique allows us to see the shift in topics from those surrounding just Trump, to more of an outward focus on Joe Biden and the Presidential election.

Figure 3. Dominant topic streams for Parler posts for the entire dataset. 

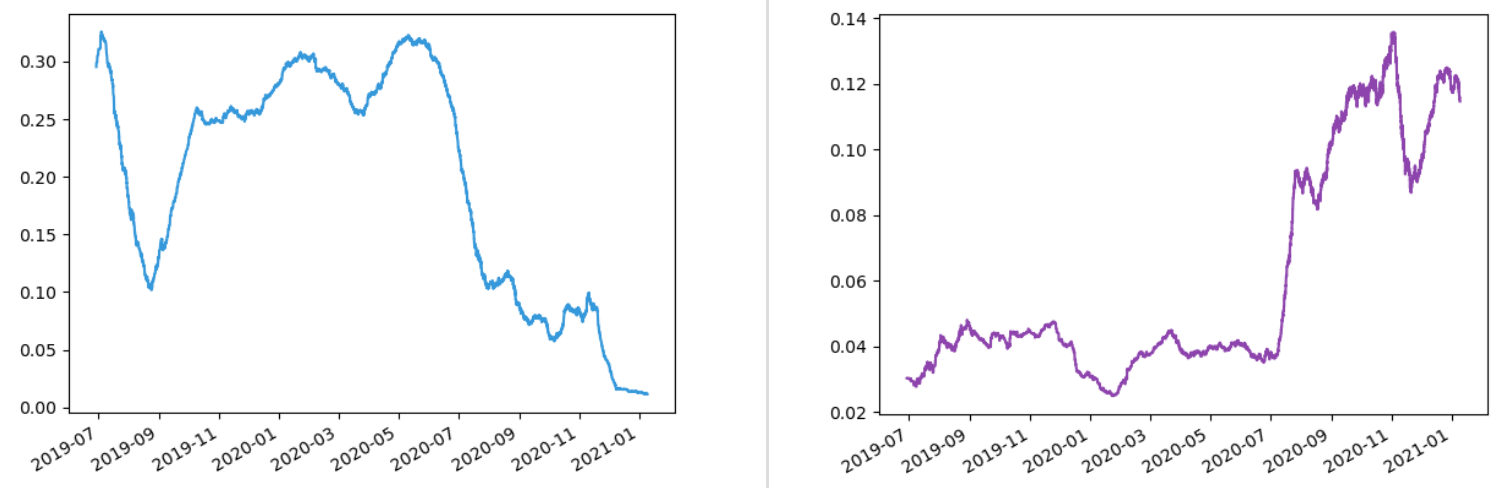

Topic 13: 'gellerreport', 'capital', 'protests',

Topic 15: 'campaign', 'biden',

'mcenany', 'kayleigh', 'cuomo'

'thegatewaypundit', 'trump', 'president'

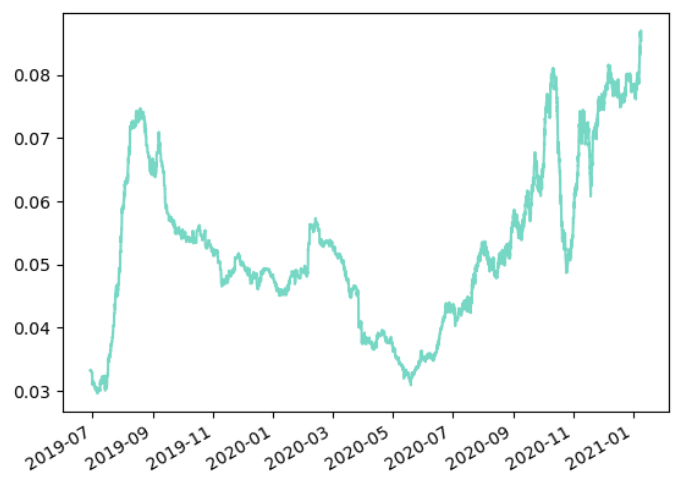

Topic 0: 'trump', 'president', 'georgia',

'supporters', 'pelosi', 'house'

Figure 4 reveals the dominant topics within the Twitter discourse for our entire dataset. Throughout 2016 and nearing the end of 2017, the conversations were dominated by combinations of Biden and Trump (Topic 1), which picked back up again in late 2018. The topics were dominated by discussions of the FBI and the election (Topic 8) in mid-2018. Topics related to race (Topic 6) became dominant in early 2019 and continued throughout 2021.

Figure 4. Dominant topic streams for Twitter posts for the entire dataset. 

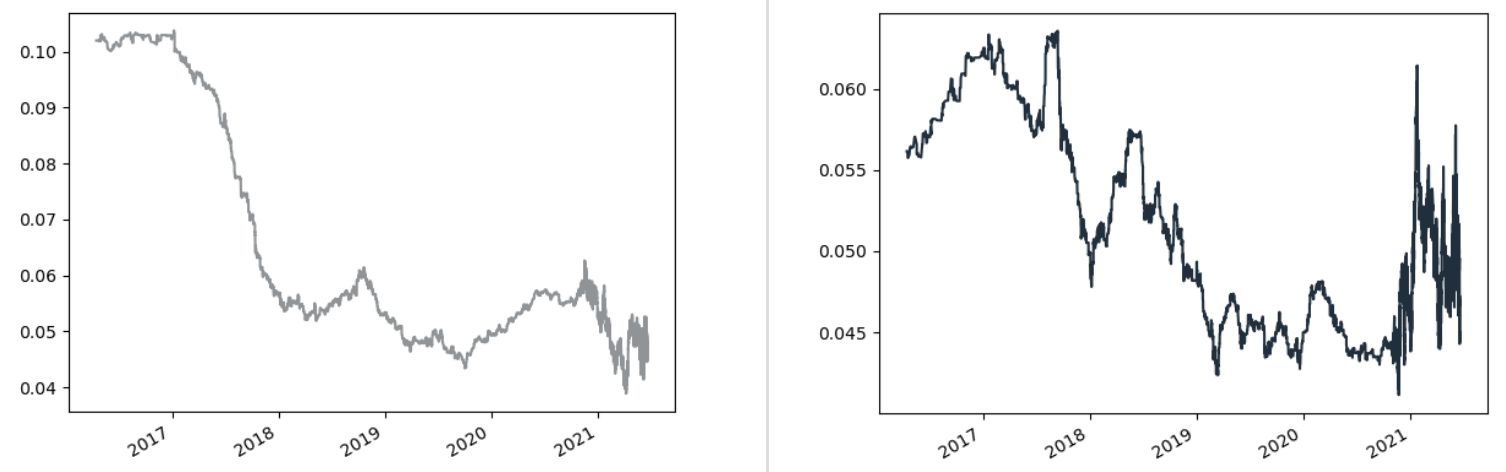

Topic 1: 'biden', 'trump', 'january', 'dumb', 'law' Topic 8: 'fbi', 'party', 'remember', 'election'

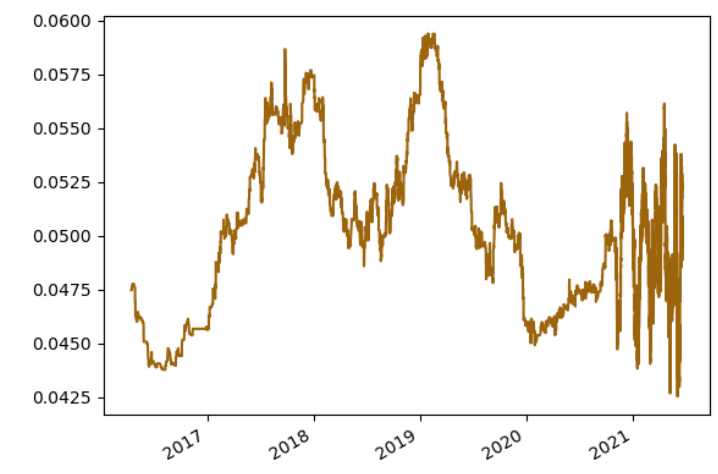

Topic 6: 'true', 'racist', 'right', 'white', 'person'

Figure 5 reveals the dominant topics within the YouTube videos based on video titles for our entire dataset. From early 2016 to late 2018 the topics were dominated by combinations of Trump and unity (Topic 0). These were usurped briefly in early 2018 by combinations of ideas of 'white guilt' and a 'great order' (Topic 3). The dominance of combinations of discussions about Biden, 'president', and 'insanity' (Topic 13) emerged from the group beginning in late 2020 and lasting through early 2021. Conversations about racism (Topic 9) also became dominant in early 2021.

Figure 5. Dominant topic streams for YouTube videos based on video title for the entire dataset. 

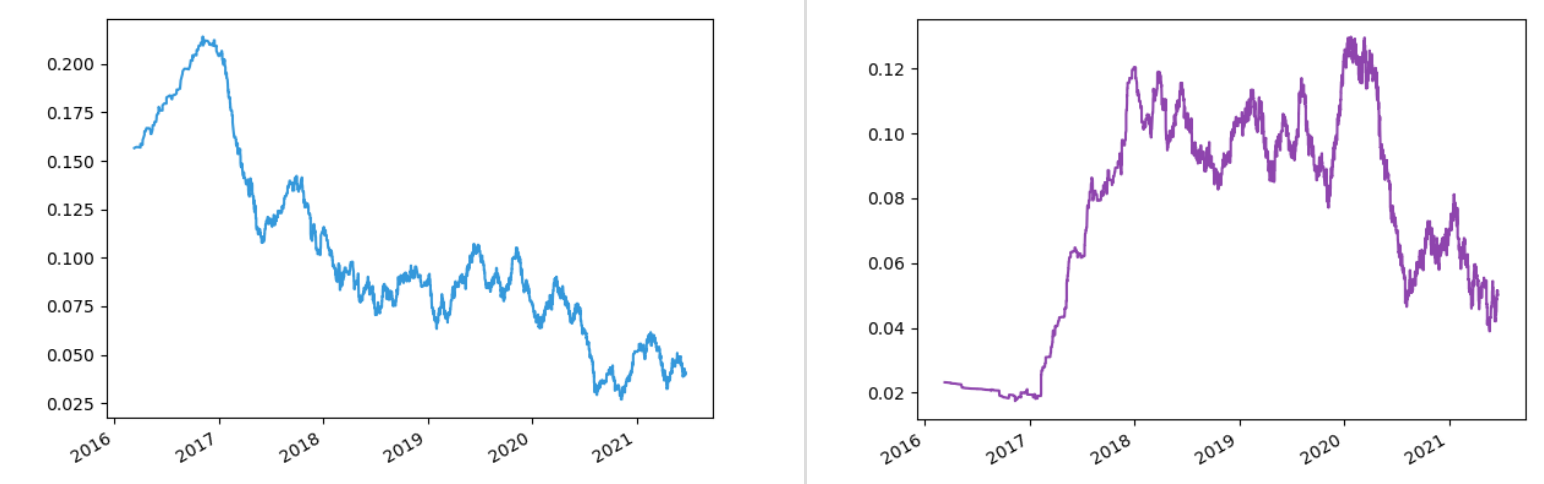

Topic 0: 'crowder', 'unity', 'shapiro', 'trump'

Topic 3: 'great', 'order', 'white', 'guilt', 'party'
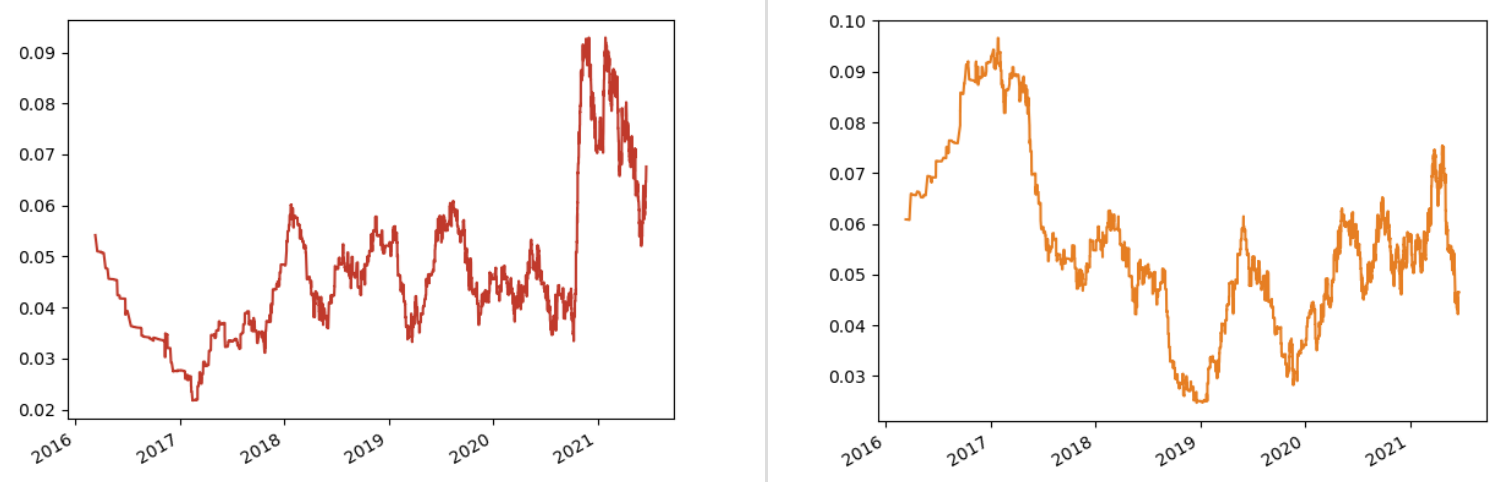

Topic 13: 'biden', 'president', 'media', 'advice', 'world', 'insanity', 'press'

Topic 9: 'mugclub', 'crowder', 'racism', 'bill', 'biden', 'cancelled', 'derek'

Figure 6 reveals the dominant topics within the YouTube related videos (those recommended to viewers) based on the video title for the entire dataset. Videos by Ben Shapiro (Topic 18) and Matt Walsh (Topic 16) seemed to have been gaining dominance over one another throughout the entire timeframe of the dataset. Aside from these, starting in around late-2016 and remaining dominant through 2021, we see combinations of conversations about Biden, Putin, and gun control (Topic 10). Then dominance shifts to combinations of racism, hate, whiteness, and privilege (Topic 19), peaking in early 2018, but remaining dominant until late 2018.

Figure 6. Dominant topic streams for YouTube related videos (recommended to viewers) for the entire dataset. 

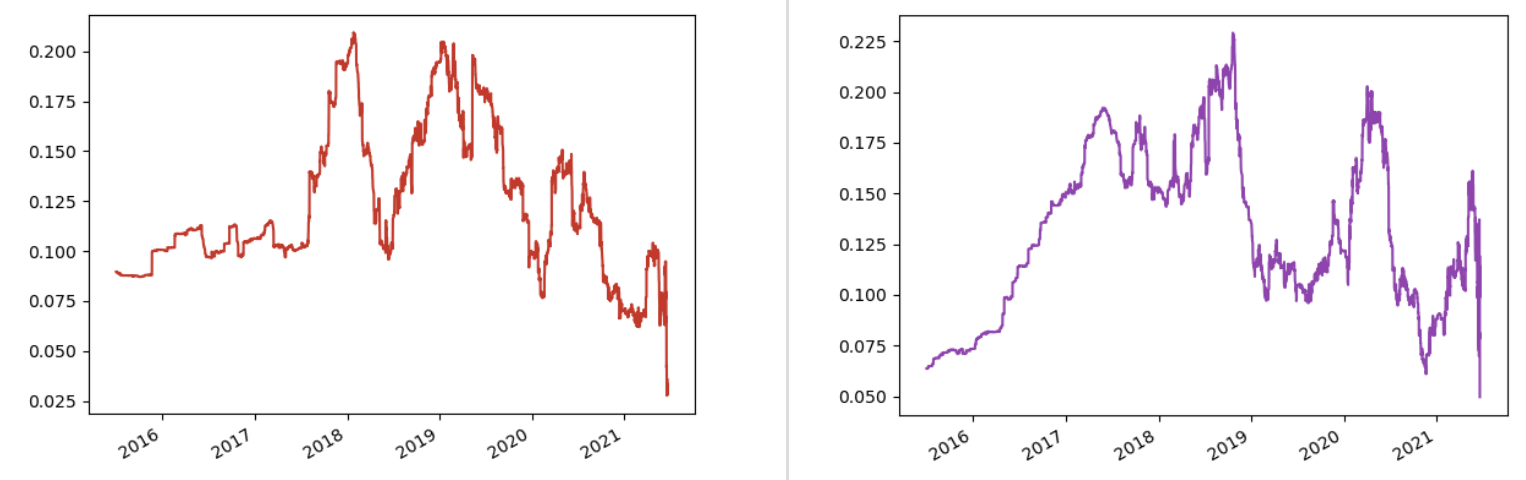

Topic 18: 'shapiro', 'bbc', 'mayor', 'wrecks',

Topic 16: 'walsh', 'crowder', 'training', 'war', 'navy' 'explores', 'bisexual'
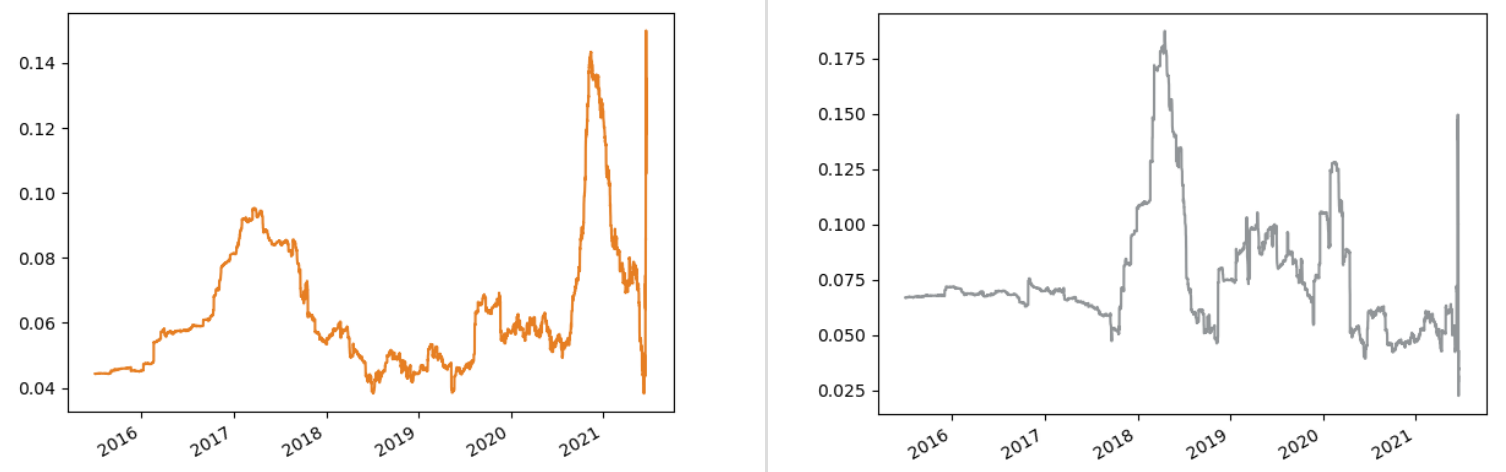

Topic 10: 'biden', 'anti', 'putin', 'trump', Topic 19: 'racist', 'hate', 'whiteness', 'privilege', 'showdown', 'control', 'gun', 'donald', 'cnn' 'male', 'falsely', 'labeled'

\section{Analysis of Topic Streams Surrounding the January 6, 2021, United States Capitol Riot}

We reduced each of our original datasets down to the timeframe of November 1, 2020, to March 1, 2021, and re-ran our topic streams analysis containing the LDA topic modeling methodology in order to discover if discourse significantly changed after the election.

Although the January 10, 2021, suspension of Parler prevented our analysis of topics beyond that point, we see that the primary discussion topics in this dataset highlighted 
the discourse around Trump and his failed election. Topics such as Georgia ballots, Supreme Court, and voter fraud dominated the discussion during this time frame (Topics 3, 0, 18). Around the end of December 2020, the topics began to integrate discussions on the newly elected President Joe Biden and lockdowns due to COVID-19 (Topic 10) and some sets of text began to discuss the idea of the election having been stolen and calls for rallies.

Figure 7. Dominant topic streams for Parler posts for the two months prior and post the January 6, 2021, U.S. Capitol riot (November 1, 2020 to March 1, 2021).
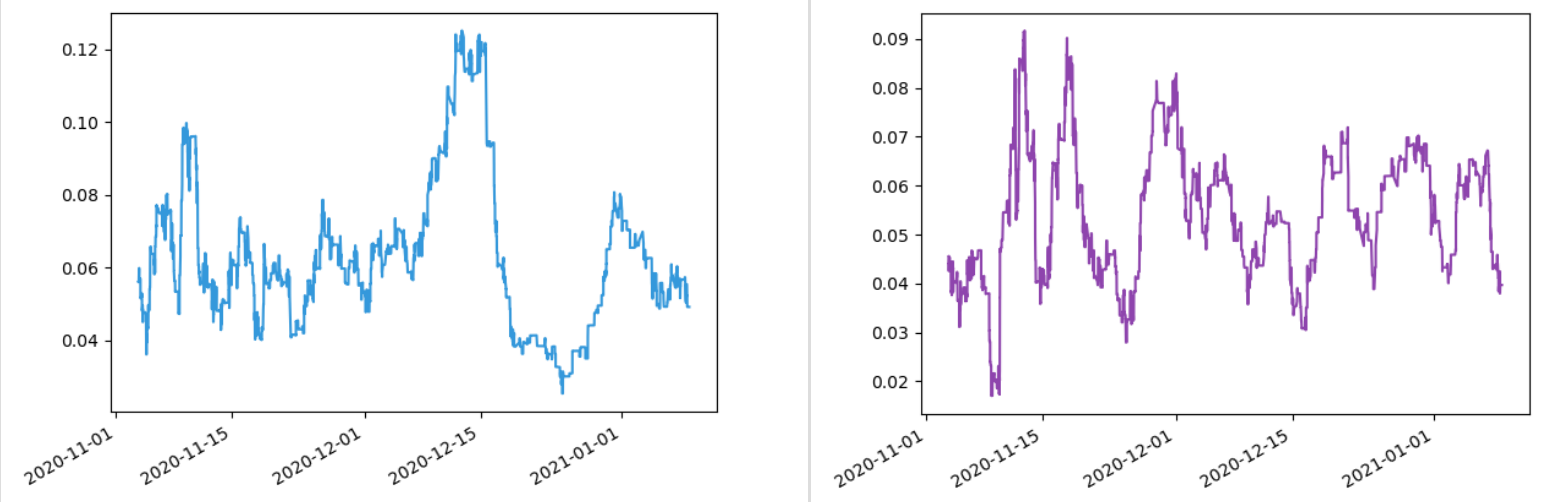

Topic 3: 'trump', 'election', 'georgia', 'ballots', 'court', 'pennsylvania', 'fraud'

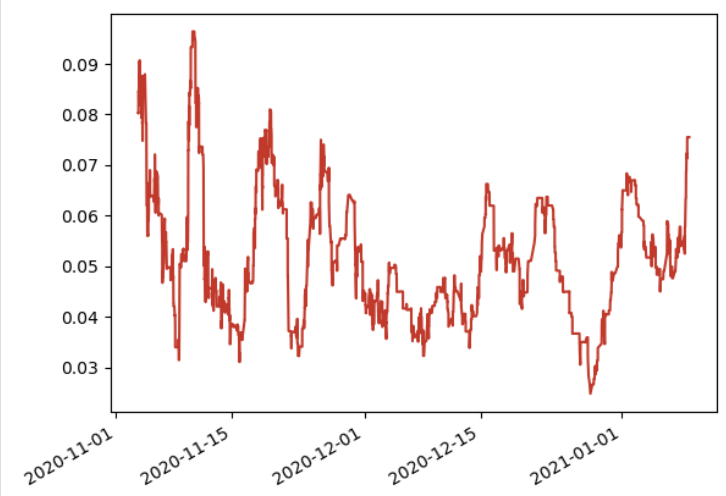

Topic 10: 'lockdown', 'china', 'covid', 'trump', 'biden', 'new', 'president', 'election'
Topic 0: 'election', 'trump', 'votes', 'biden', 'georgia', 'fraudulent', 'arizona'

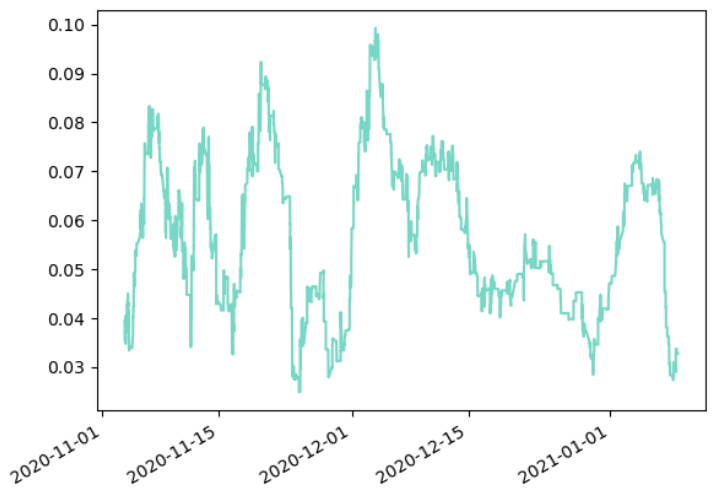
'breaking', 'country', 'court', 'covid', 'trump' 
Figure 8 reveals the dominant topics within the YouTube videos based on video titles for the two months surrounding the January 6, 2021, Capitol riot, when the conversations shifted to combinations about Biden and race, specifically the idea of 'white guilt', and the use of the terms 'going', 'free', and 'order' (Topic 8, 2, and 0). The pre-event conversation combinations also included those about Trump, impeachment, democrats, election, news, and 'covid' (Topic 3). Soon after the riot event, however, the discussion about 'white guilt' was amplified (Topic 0), and the conversations about Biden (Topic 8) remained steady.

Figure 8. Dominant topic streams for YouTube videos (based on title) for the two months prior and post the January 6, 2021, U.S. Capitol riot (November 1, 2020, to March 1, 2021).

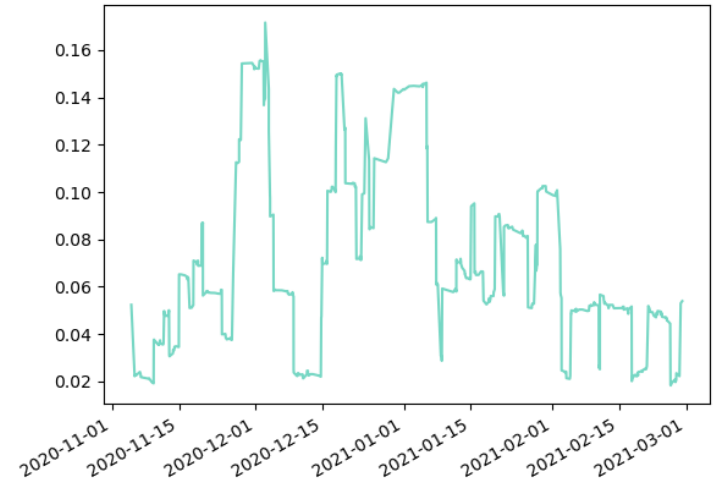

Topic 8: 'biden', 'white', 'free', 'order', 'party', 'great', 'guilt', 'roll', 'thanksgiving', 'special'

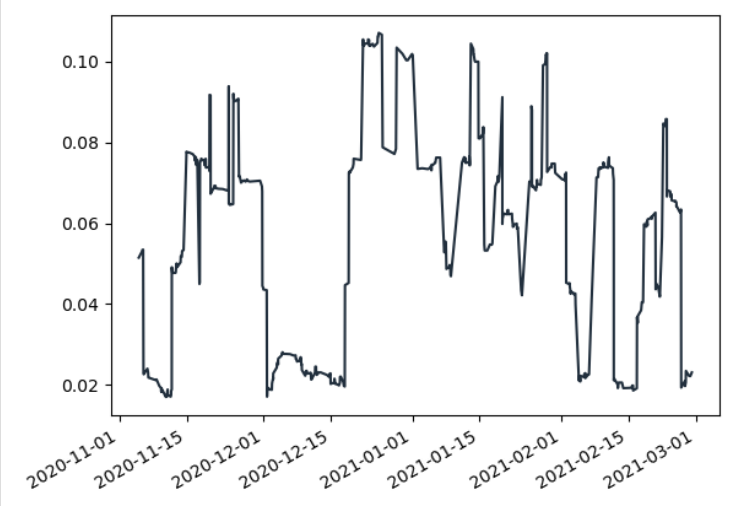

Topic 2: 'going', 'free', 'guilt', 'white', 'children', 'public', 'antiwhite', 'biden', 'back', 'health'

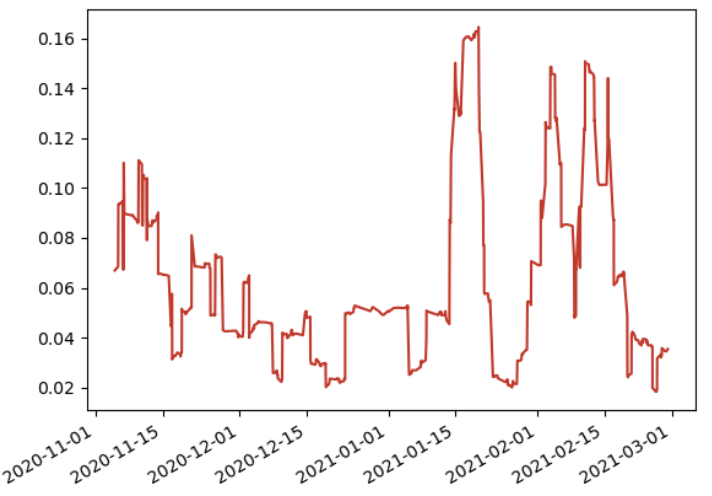


Topic 0: 'white', 'election', 'guilt', 'people', 'going', 'free', 'media', 'biden', 'calls', 'mugclub'
Topic 3: 'trump', 'impeachment', 'insane', 'democrats', 'election', 'news', 'new', 'covid'

Figure 9 reveals the dominant topics within the YouTube related videos (based on video titles) that were recommended to viewers during the two months surrounding the January 6, 2021, Capitol riot. Leading up to the event, the topics were dominated by combinations of Biden, Trump, Michigan, 'covid', the CDC, and ideas about their being 'liars' and an 'agenda' in the mix (Topic 6). Soon after the event, the topic shifted to a 'racist' 'women' 'tiktoker' (Topic 10). This topic exhibits characteristics of virality, which may have been a deliberate attempt to distract attention from topics about the police (Topic 14), which also gained traction soon after the event.

Figure 9. Dominant topic streams for YouTube related videos (recommended to viewers, based on video title) for the two months prior and post the January 6, 2021, U.S. Capitol riot (November 1, 2020, to March 1, 2021).

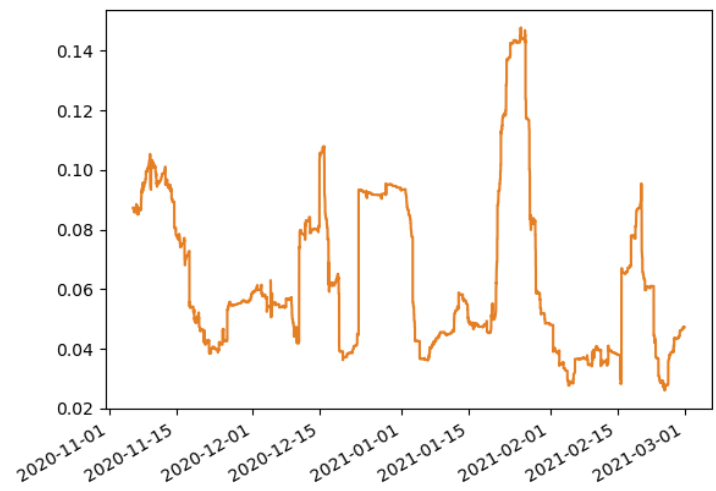

Topic 6: 'biden', 'donald', 'win', 'agenda', 'michigan', 'covid', 'asinine', 'liars', 'cdc'

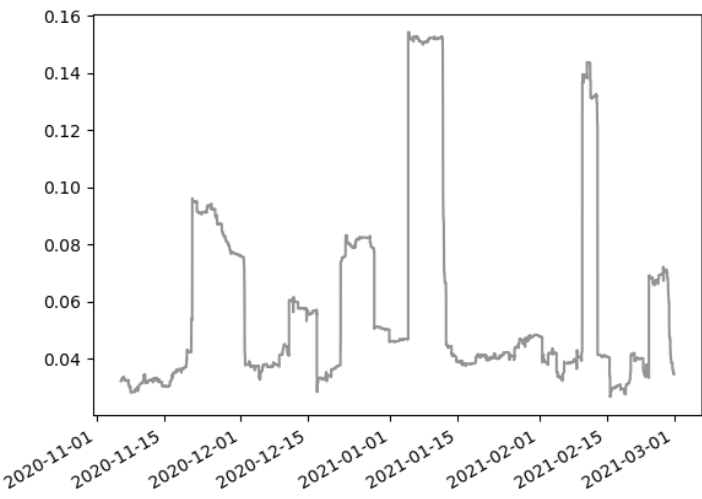

Topic 10: 'people', 'family', 'says', 'racist', 'awomen', 'tiktoker', 'hollow' 


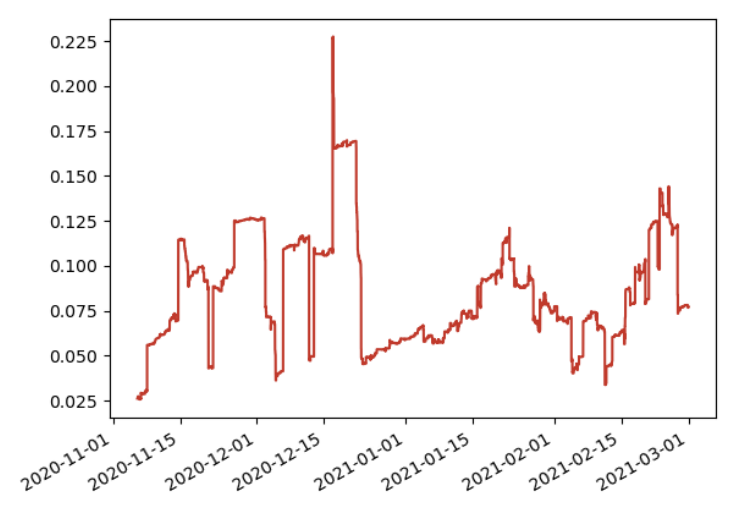

Topic 14: 'shapiro', 'police', 'podcast', 'talks', 'right', 'reacts', 'woke'

The topic modeling analysis revealed the unique nature of conversations that take place on each distinct platform. Topics related to general daily life dominated discourse on both Twitter and YouTube. Parler discourse, on the other hand, remained almost exclusively political in nature, intensifying around January 6 on issues of voter fraud and 'stealing the election.' Twitter users campaigned to get others to move to the alternative platform Gab and became particularly interested in topics related to Russia. Shortly after the Capitol riots however, the focus on Twitter became more philosophical (man, God, world). Titles of YouTube videos both pre- and post-event were political in nature with an emphasis on race. Leading up to the event, however, a greater focus on Trump, impeachment, and COVID-19 were identified. After the event, an amplification of the topics of 'white guilt' and Biden can be seen. Within the titles of the YouTube related videos (those that are recommended to viewers), topics are political in nature leading up to the event, with hints of conspiracy theories (Biden, Trump, Michigan, covid, CDC, liars, agenda). Next, we turn our focus to studying our second objective; to assess the reach, influence, and coordination amongst far-right and extremist groups operating on these platforms.

\subsection{YouTube Channel Engagement Statistics}

In this section, we discuss our examination of the user engagement statistics of our six seed YouTube channels. These include the subscribers and views that each channel gained monthly. Looking at these trends allows us to determine whether there was an obvious increase or decrease in reach of these alt-right actors both leading up to and following the January 6, 2021, U.S. Capitol riot event. One of our seed channels was 
terminated (Lauren Witzke) by YouTube soon after our initial data collection. Due to this cancellation, we were unable to retrieve engagement statistics for this channel. The other five channels bear the titles: Ben Shapiro, John Doyle, Matt Walsh, No White Guilt, and StevenCrowder.

As can be seen from Figure 10, Ben Shapiro and Matt Walsh both registered an increase in subscribers and views surrounding the January 6, 2021, event, suggesting these channels had an amplified influence on viewers at this time. John Doyle and No White Guilt, on the other hand, experienced a decline in both subscribers and views. Finally, StevenCrowder appears to have remained steady in terms of subscribers surrounding the event, while simultaneously experiencing a slight decline in views. The figure also includes some visual evidence for the deletion of the Lauren Witzke channel.

Figure 10. Engagement statistics for our seed YouTube channels from August of 2018 to August 2021.

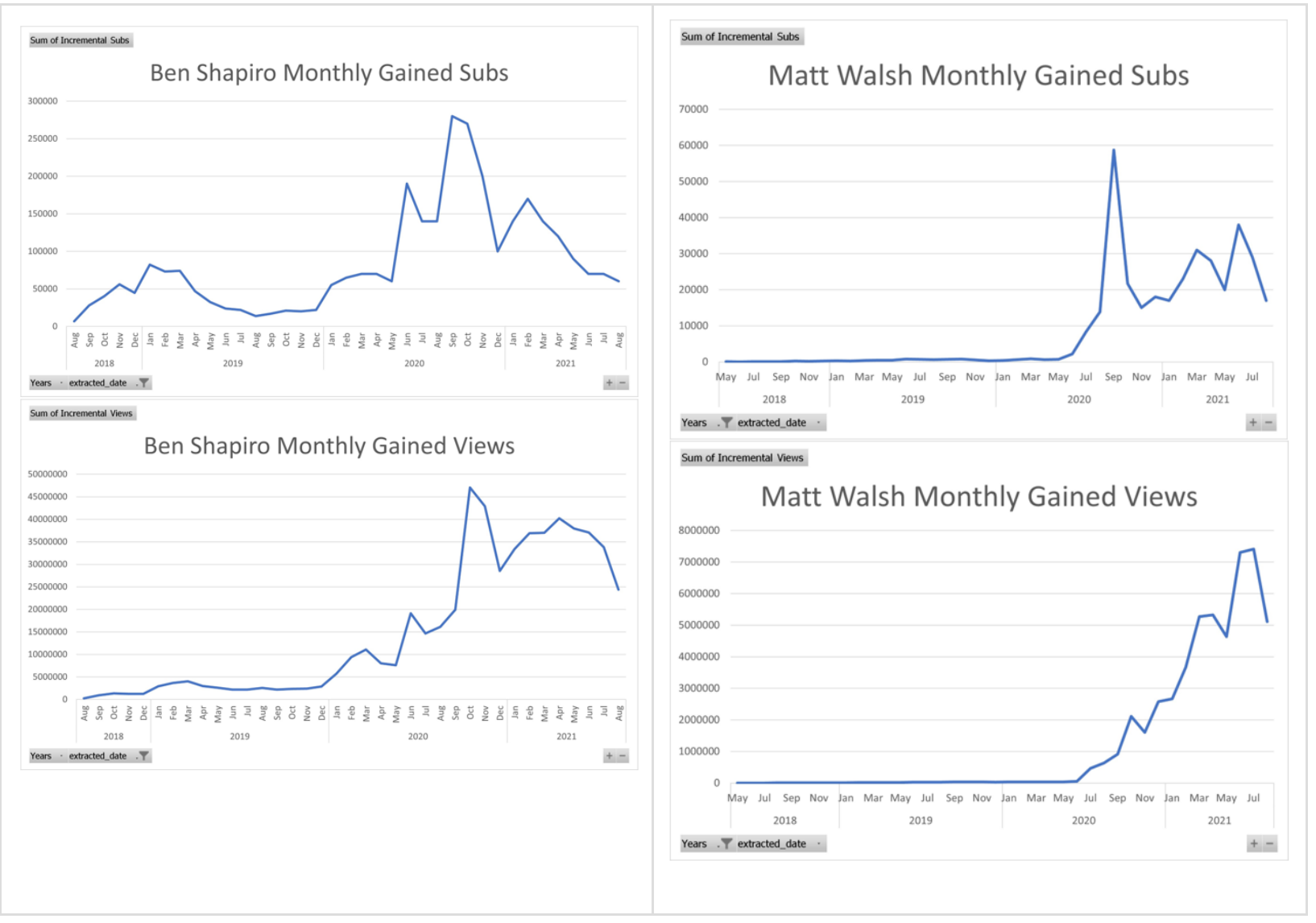




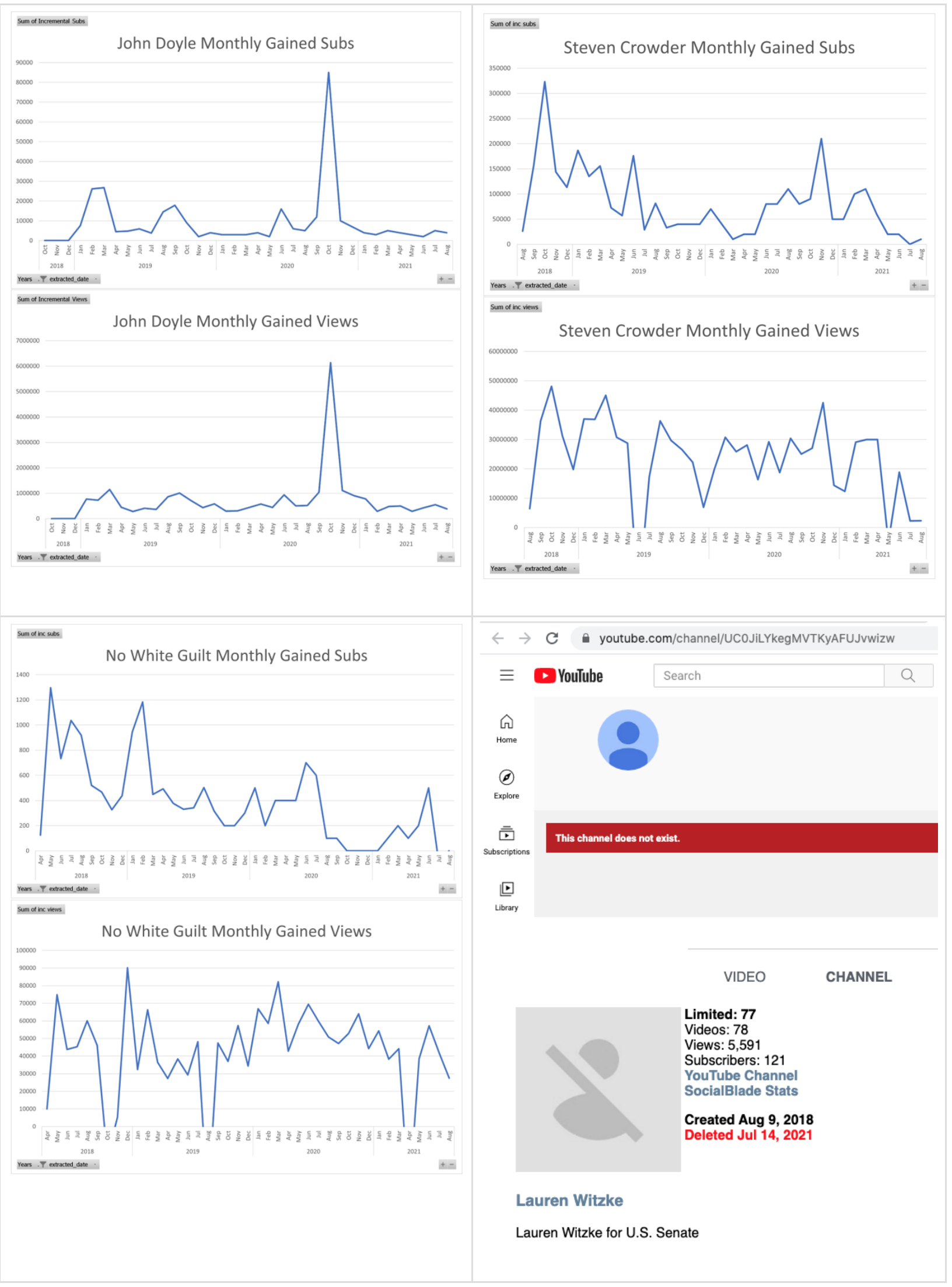




\subsection{Social Network Analysis}

We analyzed data from Parler, Twitter, and YouTube (see Table 1) to gain a better understanding of the information environment within our dataset. Social network analysis was used to identify the key actors, communities of interest, URLs that were disseminated, and to find sub-groups that shared similar ideologies within our dataset. These included user-mention, user-URL, and user-hashtag networks.

\subsection{Twitter Mention Networks}

Our analysis of Twitter interactions shows the people or groups who were at the center of the conversations among users, as well as the key actors generating the most mentions. Due to the size of the dataset, we focused our attention on groups of users that shared at least ten connections between them. We identify three distinct user communities, with the largest primarily dominated by conservative politicians and support staff, while the second largest is a community of individual and media advocates with generally right leaning ideologies, followed by a smaller group of majority democratic and left leaning media and including the (then) newly elected President Biden. We identified three key users with high levels of tweet activity that were brokering most of the mention network (Figure 11). Ali Alexander (@ali) is the man behind the \#stopthesteal movement with the most mentions by his account. The account with the second most outward mentions is @jack, the account of Jack Posobiec. He is a right-wing activist who gained notoriety after being retweeted by Donald Trump. The account with the third highest mention activity was the @FBI account. The community around the FBI account was centered on investigations into Antifa, BLM, and investigations around the \#StopTheSteal movement. These users appeared to mobilize small clusters of users between them and one other hub to broker messaging between each of their core groups, as marked by red circles in Figure 12. There was also one central user to all three core hubs. These examples of using coordinated groups of users are one tactic that groups use to amplify their messaging.

Figure 11. Twitter mentions in top communities for the two months prior and week after the January 6, 2021, U.S. Capitol riot (November 1, 2020 to January 8, 2021) showing who is at the center of attention being mentioned. 


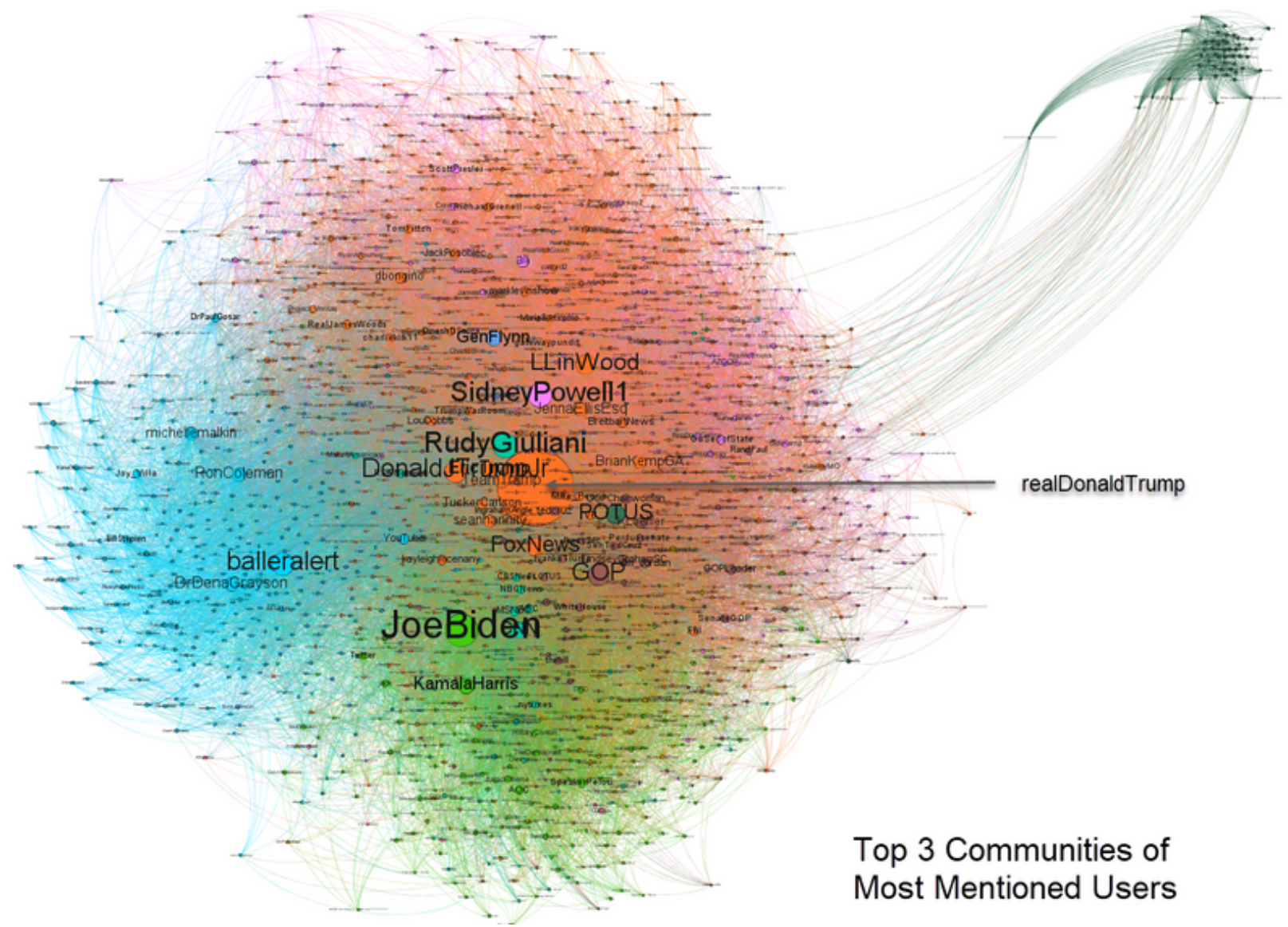

Figure 12. Twitter mentions in top communities for the two months prior and week after the January 6, 2021, U.S. Capitol riot (November 1, 2020, to January 8, 2021) showing coordination of users who are communicating the most. 


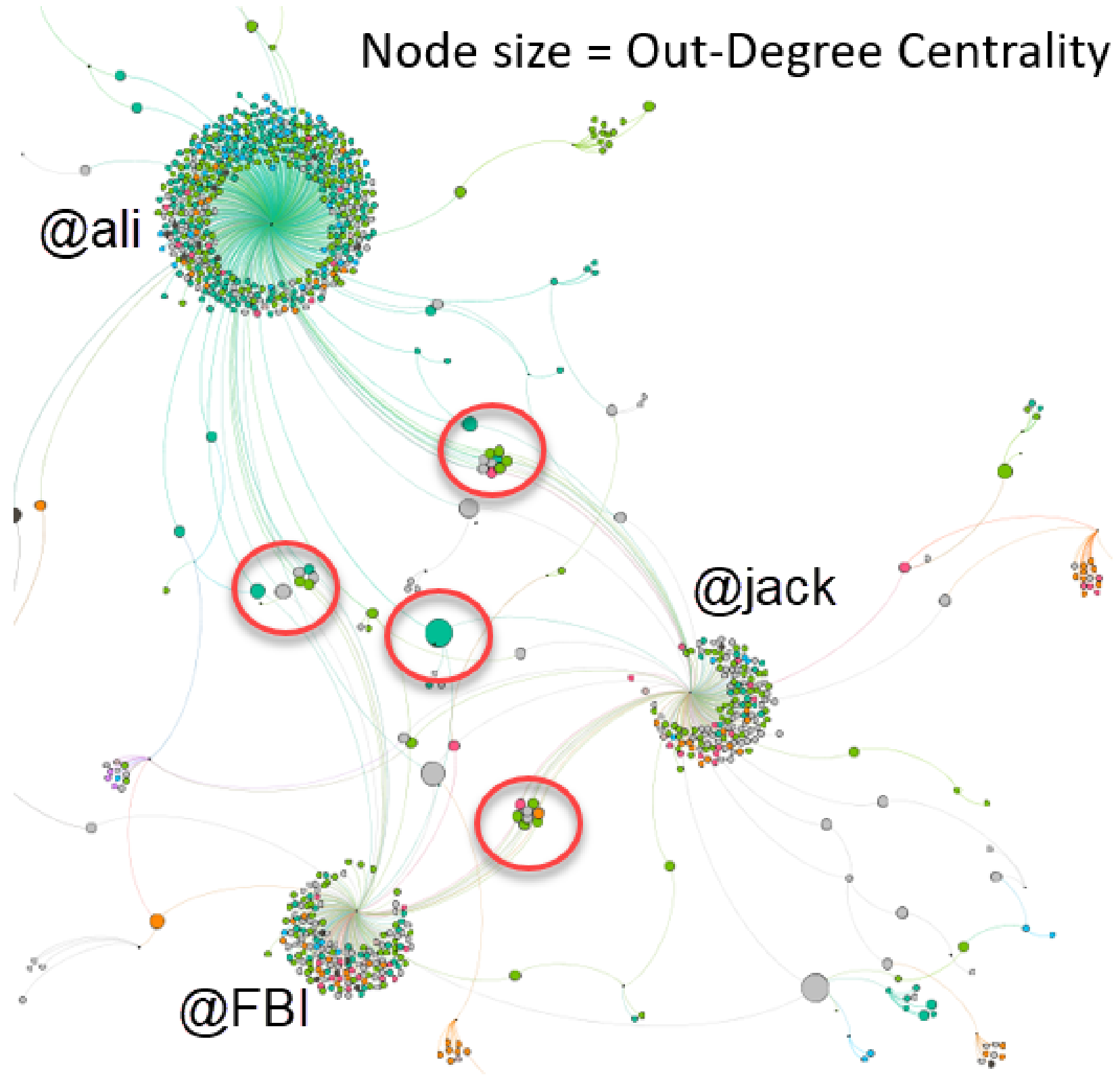

\subsection{Twitter URL Network}

We analyzed the Twitter URL network by examining which URLs were most widely shared, and by whom. The URLs most widely shared on Twitter were primarily photos and videos by other Twitter users, followed by cross-platform posts to the video platform Periscope, and then YouTube. There were 2,267 tweets pointing users to the Periscope platform which hosted a variety of videos related to 'Stop the Steal' rallies from different towns as well as users posting about patriotism. The largest number of such tweets were pointing to rallies in Colorado, two rallies in Arizona, followed by a 
rally in Beverly Hills. A complete list is provided in Table 3. This finding shows the use of cross-platform tactics by the actors for mobilization and coordination.

Table 3: Top Periscope videos shared from Twitter platform for the two months prior and week after the January 6, 2021, U.S. Capitol riot (November 1, 2020, to January 8, 2021).

\begin{tabular}{|l|l|}
\hline Periscope video title & Number of times the link was tweeted \\
\hline More \#stopthesteal Colorado Springs & 698 \\
\hline \#stopthesteal Colorado a Springs & 325 \\
\hline \#stopthesteal \#arizonahearing now! & 280 \\
\hline stopthesteal continued & 245 \\
\hline \#stopthesteal Beverly Hills & 94 \\
\hline $\begin{array}{l}\text { This ain’t time for pancakes...it's time for } \\
\text { patriotism! }\end{array}$ & 85 \\
\hline \#WYNK - S. Ct. \#stopthesteal & 23 \\
\hline $\begin{array}{l}\text { NEW SONG PREMIERE @ 8EST! “Flip it” } \\
\text { \#stopthesteal }\end{array}$ & 8 \\
\hline STOP THE STEAL - Atlanta, GA & 8 \\
\hline
\end{tabular}

The graph in Figure 13 shows which users were most active in disseminating content. At least one of the top 5 user accounts from our dataset was suspended and previously identified as a bot. A bot is an agent that communicates autonomously on social media, often with the task of influencing the course of discussion and/or the opinions of its readers. These bots can be scheduled to perform tasks on behalf of its users (Agarwal et al., 2017). Although this tactic is not unique to the right, it shows tactics used to possibly amplify content.

Figure 13: Largest community of users disseminating URLs on Twitter. Larger labels show users with the most tweeting activity for URLs for the two months prior and week after the January 6, 2021, U.S. Capitol riot (November 1, 2020, to January 8, 2021). 


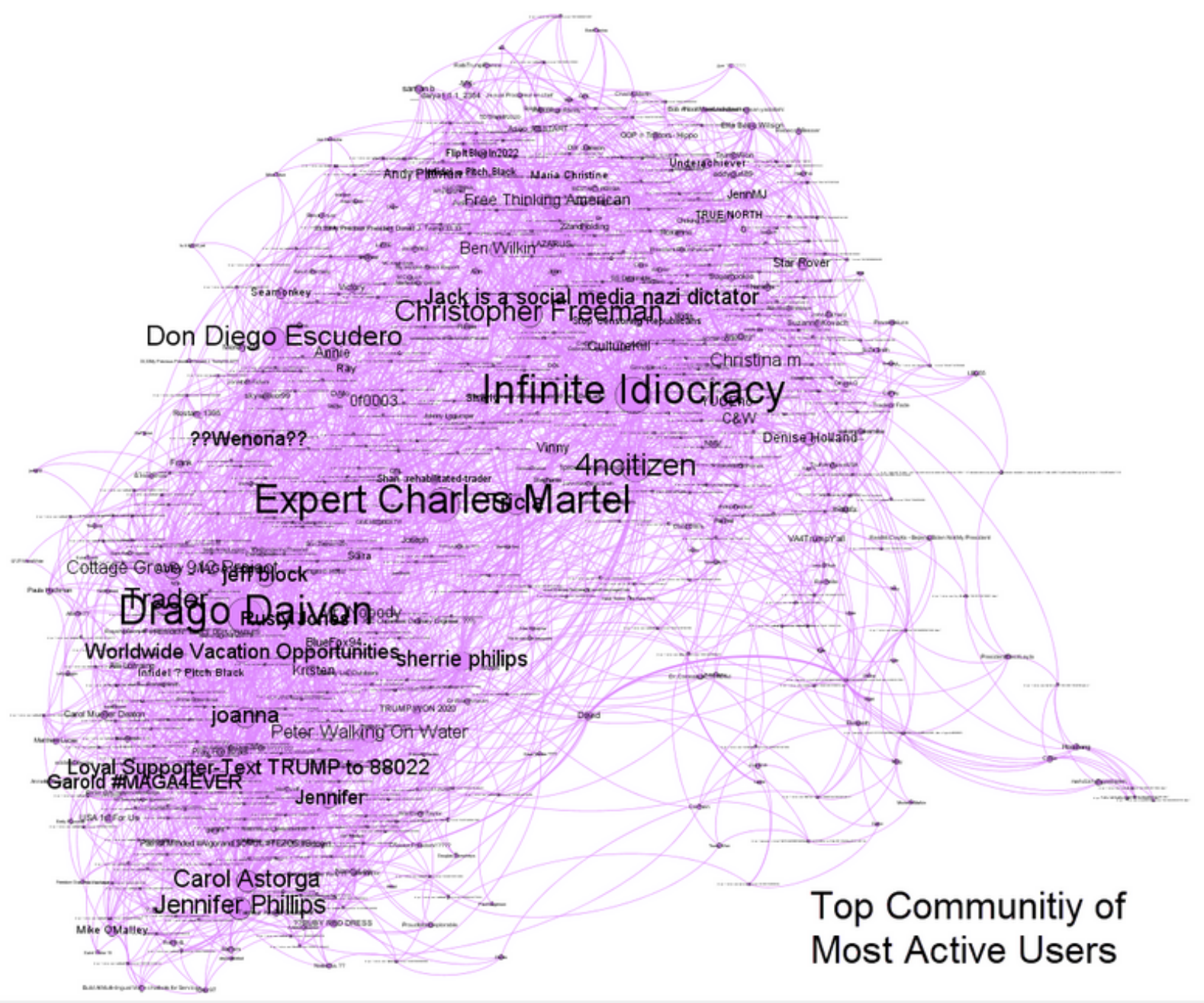

Figure 14: Twitter account screenshots for users with the most tweeting activity for URLs for the two months prior and week after the January 6, 2021, U.S. Capitol riot (November 1, 2020, to January 8, 2021). Shown below are Expert Charles Martel, Drago Daivon, and 4ncitizen from left to right. 


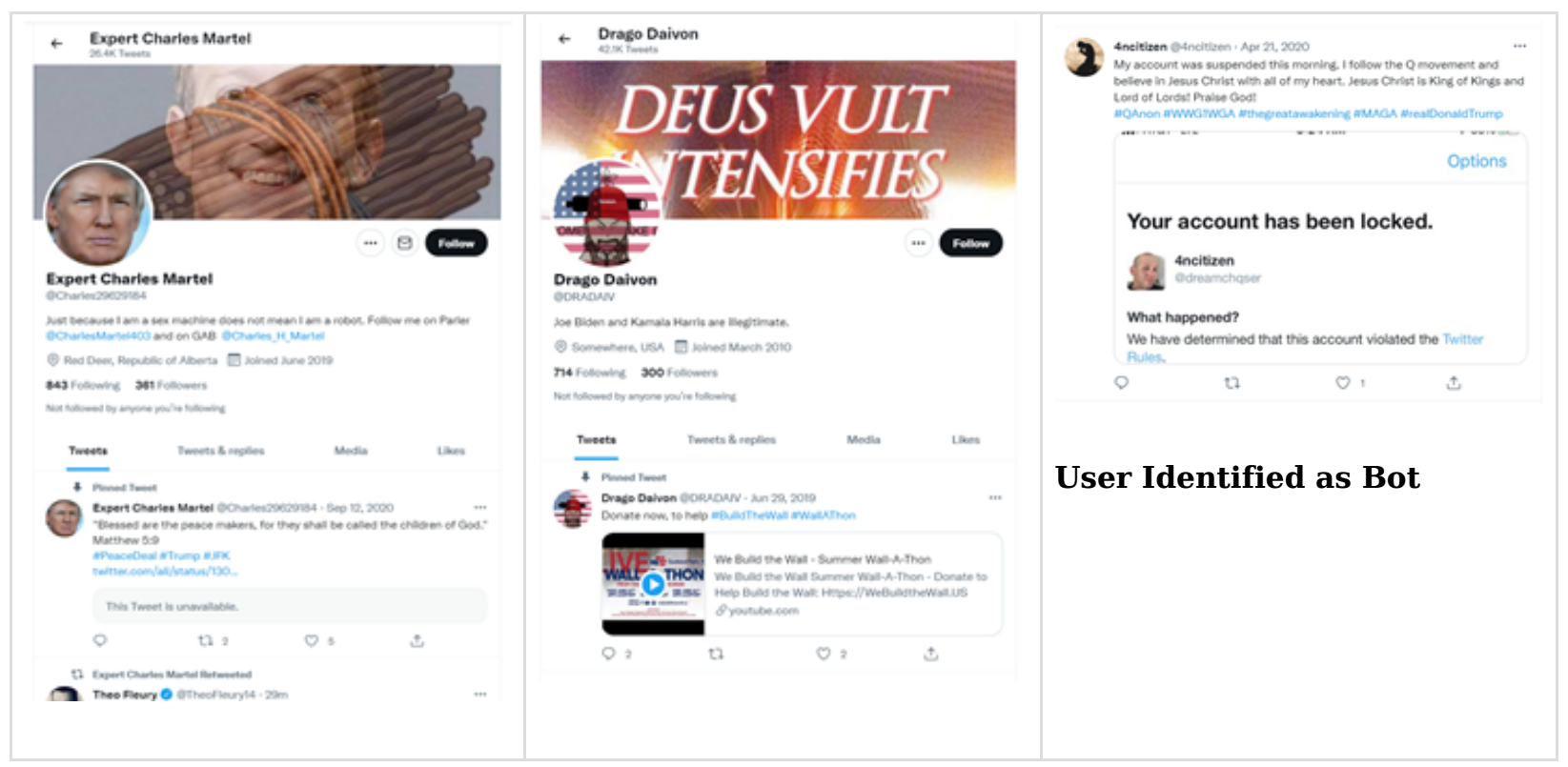

\subsection{Twitter Hashtag Network}

Hashtags used by members of the alt-right dataset followed most of the obvious political and extremist interests of alt-right actors (Figure 15). However, when we look at the expanded dataset of users, we see users who included recent social movements such as \#blm and \#georgefloyd in their tweets as shown in Communities 3 and 4 in Table 4. Users from those communities have created polarized counter messaging to further amplify the social movement (Table 4). For example, we see the 2nd largest hashtag group advocating for lockdowns, wearing masks, and social distancing. The 3rd and 4th communities consist mostly of black lives matter, antifa, protests, and calls to defund the police. In all, 41,949 distinct hashtags were detected and shared over 1 million times.

Table 4: Top 15 hashtags from each of the largest 5 communities for the two months prior and week after the January 6, 2021, U.S. Capitol riot (November 1, 2020, to January 8, 2021). 


\begin{tabular}{|c|c|c|c|c|c|c|c|c|c|}
\hline \multicolumn{2}{|c|}{ COMMUNITY 1} & \multicolumn{2}{|c|}{ COMMUNITY 2} & \multicolumn{2}{|c|}{ COMMUNITY 3} & \multicolumn{2}{|c|}{ COMMUNITY 4} & \multicolumn{2}{|c|}{ COMMUNITY 5} \\
\hline Hashtag & \# of times shared & Hashtag & \# of times shared & Hashtag & \# of times shared & Hashtag & $\#$ of times shared & Hashtag & $\#$ of times shared \\
\hline STOPtHEsTEAI & 36150 & Coronavirus & 15778 & blm & 15896 & blackLivesMatter & 10102 & Trump & 2424 \\
\hline MAGA & 8635 & COVID19 & 6421 & Antifa & 4028 & love & 583 & Trump2020 & 1907 \\
\hline Trump2020 & 3009 & WearAMask & 3622 & blackLivesMattet & 2306 & art & 338 & MAGA & 1638 \\
\hline VOTERFRAUD & 1835 & COVID & 2254 & FakeNews & 1325 & vidasnegrasimportar & 286 & Biden & 1259 \\
\hline Election2020 & 1728 & stayhome & 2058 & defundthepolice & 270 & black & 285 & Election2020 & 1006 \\
\hline BidenCheated & 1568 & trumpVirus & 1776 & antifaterrorists & 263 & blackgirlmagic & 247 & Democrats & 572 \\
\hline Americafirst & 1477 & MaskUp & 1602 & KOZP & 244 & Twitter & 203 & ELECTION & 533 \\
\hline StopTheCheating & 1318 & stayathome & 1199 & $A C A B$ & 230 & blackownedbusiness & 200 & KamalaHarris & 529 \\
\hline MillionMAGAMarch & 1314 & Covid_19 & 1103 & DC & 226 & blacktwitter & 186 & USA & 463 \\
\hline AMACforAmerica & 1039 & staysafe & 1064 & republicans & 225 & fashion & 170 & Joebiden & 410 \\
\hline BidenCheated2020 & 995 & pandemic & 1031 & $? ? ?$ & 216 & Music & 169 & Racism & 398 \\
\hline MAGA2020 & 856 & LockDown & 810 & dcprotest & 203 & instagram & 168 & America & 361 \\
\hline Electionfraud & 831 & CORONA & 772 & Racist & 172 & blackexcellence & 165 & BidenHarris202C & 344 \\
\hline ProtectTheVote & 772 & socialdistancing & 653 & BLMPlaza & 162 & georgefloyd & 152 & VOTE & 339 \\
\hline bidencheat & 737 & WearADamnMask & 592 & DCProtests & 158 & Memes & 152 & Politics & 286 \\
\hline
\end{tabular}

Figure 15. Top Hashtags used by the groups in our dataset for the two months prior and week after the January 6, 2021, U.S. Capitol riot (November 1, 2020, to January 8, 2021). Top 5 largest communities. 


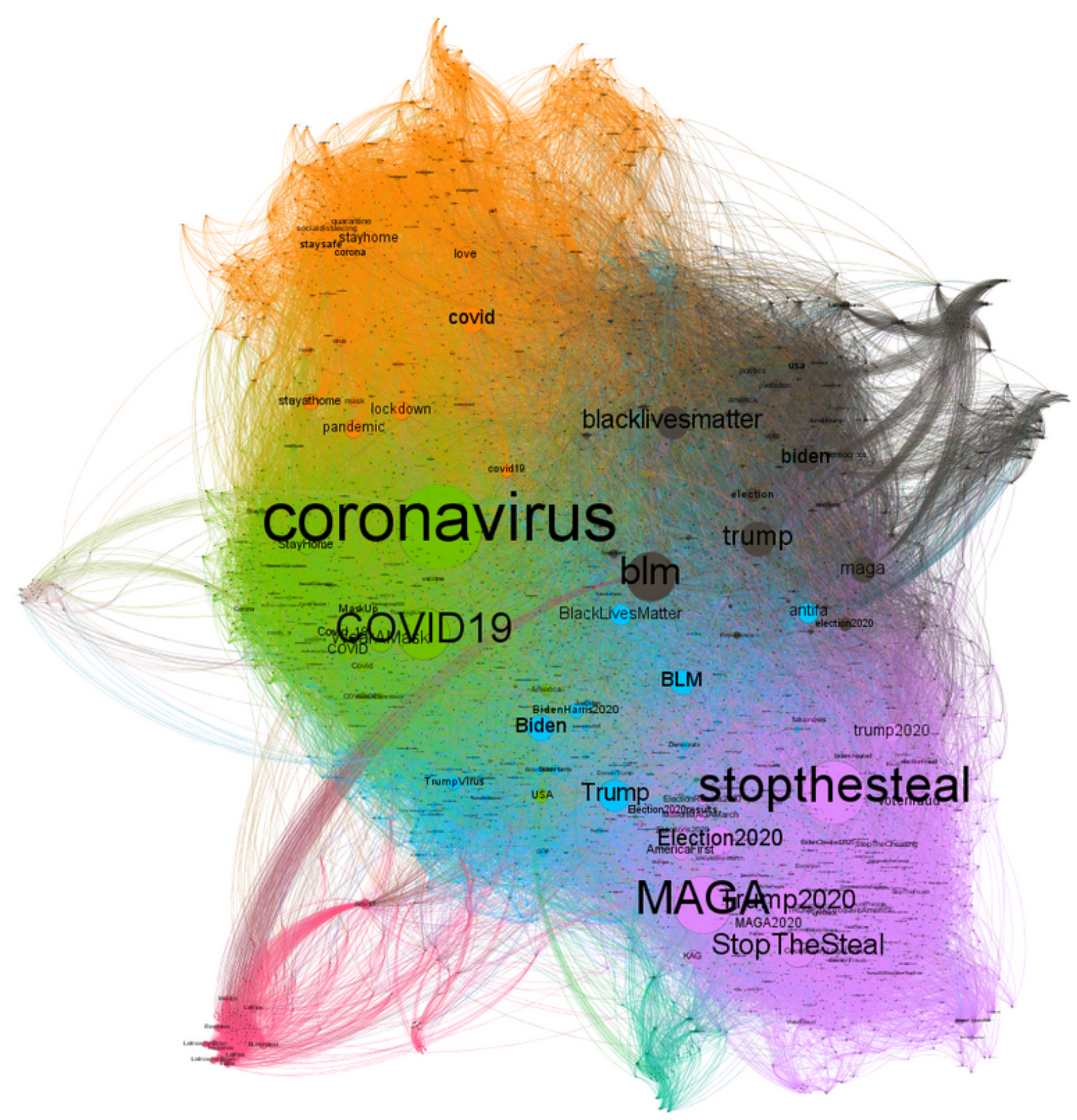

\subsection{Parler URL Network}

The users within our dataset shared a variety of URLs in their Parler posts in the two months leading up to the U.S. Capitol riots (Table 5). The top 3 URLs included links to Parler's YouTube channel and an introductory video for users on how to use Parler. The second most URL was a link directing users to the "Trump Make America Great Again Committee.' 'TMAGAC' is a joint fundraising committee composed of the participating committees of Donald J. Trump for President, Inc. ('DJTP'), Save America, and the Republican National Committee ('RNC'). These 3 URLs were shared over 2 million 
times during this time period. Parler users used the platform to push a significant amount of content to other alternative audio and video sharing platforms like Bitchute, Rumble, and Vocaroo.com. The data also revealed cross platform URL sharing to conservative supporting blog sites like Zero Hedge, Epoch Times, and the Gateway Pundit.

Interestingly, Amazon URLs were shared over 3,700 times during this time period. The most shared link was to a book about corruption in the CIA and the American government, and how the CIA has allegedly been controlling the government. There were fewer links to additional books, some self-published, about alt-right ideologies like the Deep State (mostly fiction). Other Amazon links are for Trump apparel supporting a recount or that the election was stolen. Clearly, the Parler platform was being used to push conservative ideologies. The use of cross-media and cross-platform content sharing demonstrates how these users can reach the broader public.

Table 5: Top shared URLs from Parler for two months leading up to the U.S. Capitol Riot.

\begin{tabular}{|c|c|c|}
\hline User & URL & count \\
\hline John & http://www.youtube.com/c/ParlerApp & $1,404,713$ \\
\hline TeamTrump & https://bit.ly/2YH3R9H & 678,218 \\
\hline Camillewead & https://youtu.be/TAFkineAZTs & 78,939 \\
\hline digitalmarketing101 & https://cf.trumpfriends.club & 3,625 \\
\hline ExposingClACorruption & https://www.amazon.com/dp/B07V9JT65Y & 2,850 \\
\hline $2020 w t f$ & https://www.digitalwarriorsusa.com & 1,748 \\
\hline Sonofabeavertile & https://rumble.com/vb49zb-taxajermy-maga-rock-lyric-video.html & 1,490 \\
\hline Dian5 & https://image-cdn.parler.com/5/1/51vDzVpJ4p.jpeg & 1,427 \\
\hline Dian5 & https://image-cdn.parler.com/v/t/vt6EPCR70d.jpeg & 1,426 \\
\hline LmLwLL & https://www.digitalwarriorsusa.com & 1,247 \\
\hline iAmFreedomMan & https://www.bitchute.com/video/STZjsXG2tvmV/ & 1,204 \\
\hline LoyalPatriot & https://ept.ms/DownloadApp & 1,084 \\
\hline Miked32689 & https://gofundme.com/f/help-officer-fired-for-freedom-of-speech & 968 \\
\hline presidentusa & https://rb.gy/9fwfdf & 802 \\
\hline Hwjbuffalo & https://www.foxnews.com/apps-products/index.html. & 773 \\
\hline ThomasKent & http://EverPraiseApparel.com & 757 \\
\hline ThePrivateEye & https://www.foxnews.com/apps-products/index.html. & 665 \\
\hline Schmirk74 & https://m.theepochtimes.com/video-this-fight-is-not-over-trump-attorne) & 656 \\
\hline iAmFreedomMan & https://www.bitchute.com/video/91VKfwiqH3r6/ & 636 \\
\hline
\end{tabular}




\subsection{Parler Mention Network}

We also analyzed the Parler mention network to determine who was communicating with whom (Figure 16). Two primary communication clusters stood out. The largest community consists of Trump and his strongest advocates. The second largest community was mostly conservative TV and talk show hosts, who served as bridges to the primary cluster of Trump mentions. We also identified a few suspected bot accounts in this largest Parler community which were amplifying content, as described below.

Figure 16: Parler Mention network showing users at the center of communication.

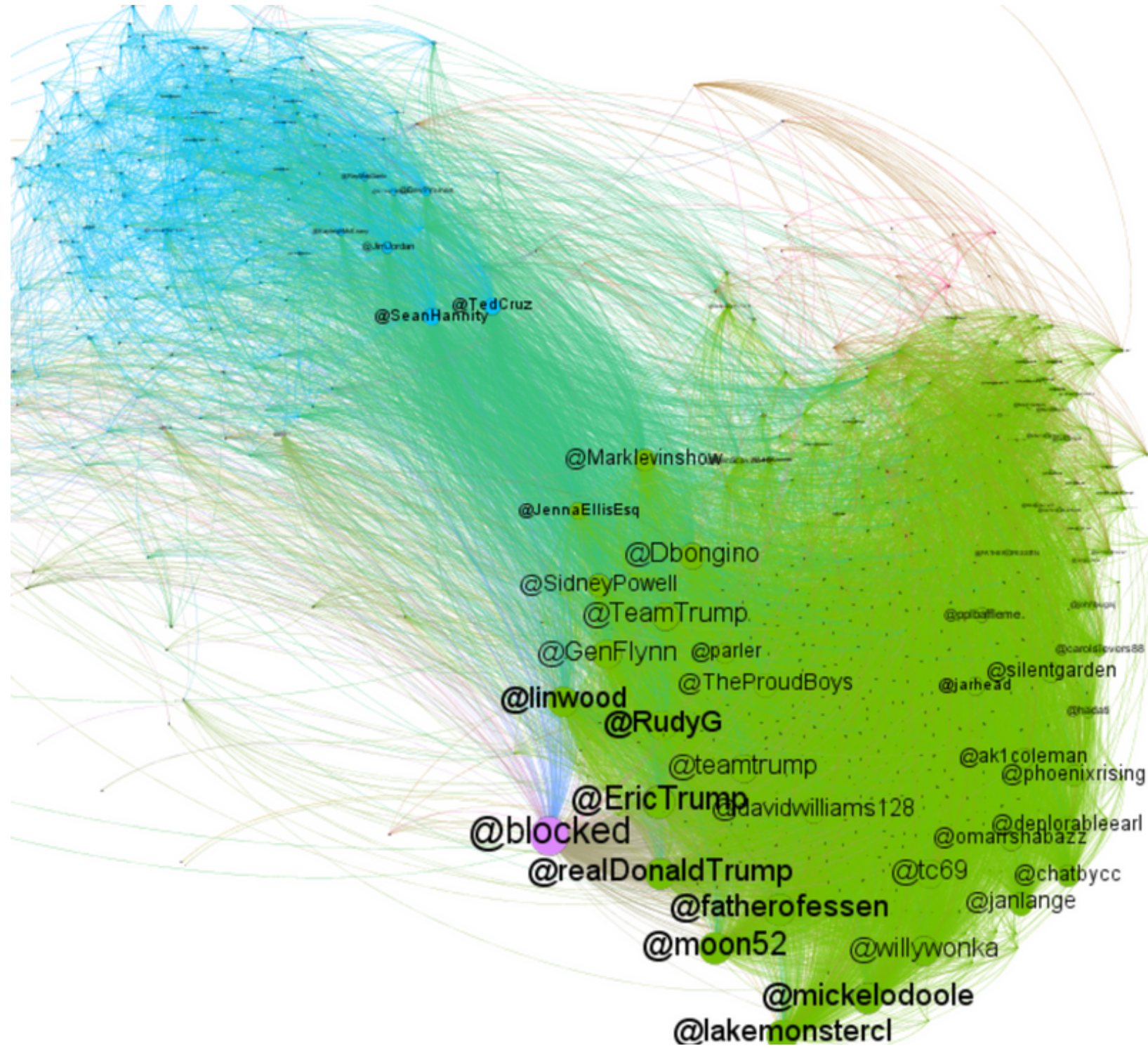


Figure 17: Parler user LakeMonsterCL user showing up on anonymizer site Nitter associated with possible Jan. 6 propaganda.

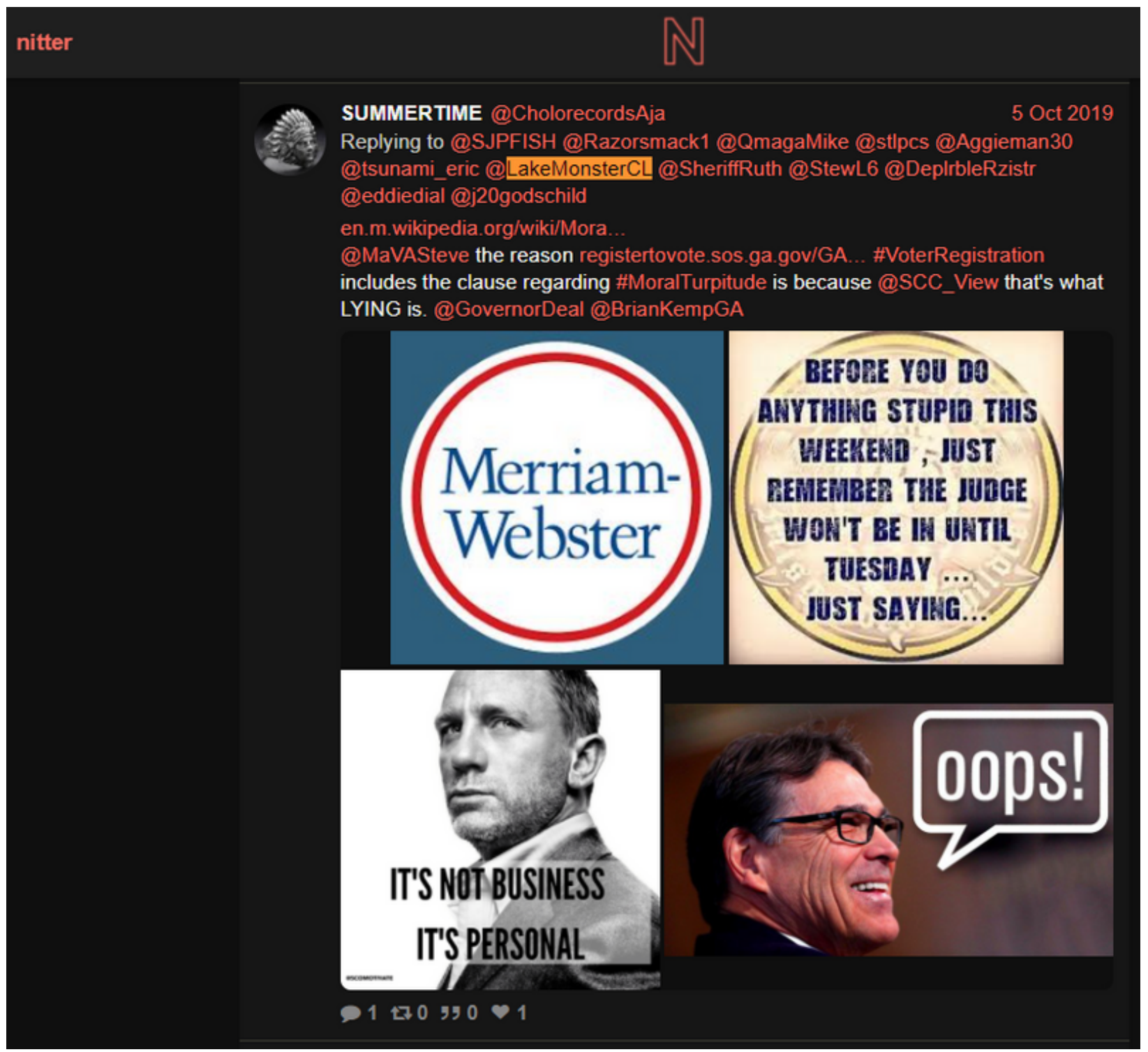

An analysis into one high activity Parler account, @LakeMonsterCL, shows this account engages in follow back groups and shares and deletes political content in high volume, characteristic of an echo-chamber effect $\underline{1}$ produced by bots. This same user account was found to cross post on Twitter. A manual Google search of this account also shows activity on the Nitter platform (Figure 17). Nitter, redirects Twitter web links to an alternative version of the social network that removes the code necessary to track your behavior (Maxwell, 2020). In other words, Nitter is a new front-end for Twitter that helps hide IP addresses or other fingerprints from advertisers or anyone else that might be tracking your activity. 
Another user, moon52, helped spread the reach on Parler by coordinating follow back activities as shown in Figure 18. This user had the highest in-degree centrality in our network, which measures how many connections this user has. This technique is another influence tactic used by members on alt-right platforms.

Figure 18: Parler snapshot of a user engaging in echo-chamber follow back tactics.

\section{Nov 9 th, 2020 \\ +98 8.9k views}

I'll help you get some followers, hopefully Here's one of the good ones Patriots Let's show him some love

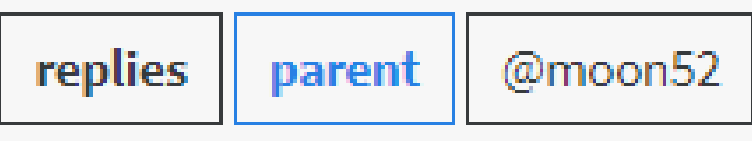

Clearly, users from the Parler network used multiple tactics to shape their conservative narratives. As expected with the Parler platform, the heaviest users were either from the Trump campaign, or strong supporters of the conservative movements. Parler was used as a tool to disseminate information across multiple platforms, including the traditional Twitter and YouTube platforms, but also new alternative voice and video platforms such as Gab, Gettr, Bitchute, and Vocaroo. Finally, Parler users appeared to engage in inorganic behaviors by using bots, anonymizers, and IP masquerading.

\section{The Conclusions and Future Work}

The key objectives of the study were to (1) identify how the alt-right shape, disseminate, and amplify their radicalized messages to the broader public using social media platforms, including so-called 'free speech' or alternative platforms; and (2) assess the reach, influence, and coordination amongst far-right and extremist groups operating on these platforms. This study utilized socio-computational methods to compare alt-right user behavior on the mainstream platforms, YouTube and Twitter, along with the alternative social media platform Parler to address the objectives. Toxicity assessment, topic stream analysis, social network analysis, and social cyber 
forensic analysis helped identify key far-right actors and the tactics used to shape, disseminate, and amplify their narratives.

More specifically, while studying the first objective, topic modeling and toxicity analysis detected patterns of highly polarized narratives having an oversized impact on public discourse among followers of these alt-right actors. Leading up to the 2021 January 6th U.S. Capitol attack, dominant narratives included Biden, Trump, Michigan, 'Covid' and the CDC, including the implication that the organization were 'liars' and had an 'agenda.' Additionally, these alt-right actors amplified these divisive narratives by engaging in growth hacking techniques such as asking their own followers to like and follow other similarly minded content creators, thus extending the network of influence exponentially. Further, our findings are consistent with Walther and McCoy (2021) which demonstrate that key public figures such as politicians or media personalities play key roles in legitimizing divisive rhetoric, disinformation, and conspiracies amongst the broader public. Considering the FBI report (2019) that warned that believers in conspiracy theories were 'very likely' to pose a national security threat, these findings offer valuable insights into how extremist groups operate within influence networks (p. 5).

Our findings for the second objective show the alt-right employed sophisticated techniques involving cross-platform coordination, deploying of cyber bots, and IP masquerading to amplify and mobilize other users within and outside these networks. We showed that Parler and Twitter were used as tools to broker messages across alternative platforms like Periscope, Gab, Bitchute, and Nitter. The use of cross-media and cross-platform content sharing demonstrates these users are attempting to influence the ideologies of the broader public.

Our work has demonstrated tactics and techniques used in traditional and alt-right speech platforms. Although we identified the prevalent use of alternative platforms, a deeper analysis of content on those platforms needs to be conducted as part of future work. Are similar techniques used in these platforms, or do we see users engage in different tactics? Another opportunity for our extended work explores the connective action components within these information campaigns. Given the number of rallies and the U.S. Capitol Riot, we know there were many physical events where users were mobilized. We would like to apply our connective action framework (Spann and Agarwal, 2021) to these alt-right platforms to differentiate between strong opinion and a call to violence. 
There were some limitations to the study worth mentioning. Firstly, most social media platforms - even those considered 'free speech' platforms - prohibit the use of hate speech and therefore creative linguistic codes and phrases have been utilized to avoid detection, making it often difficult to assess the true meaning of content. Secondly, the most ardent white supremacist extremists (WSE) were banned from many social media sites in the past several years, leaving our study to rely on peripheral actors who still have active accounts and these actors mostly fall on the mild spectrum of alt-right ideology. At the time of our study, we did not have access to data from sites such as Gab or Telegram where some of these banned actors may still have active accounts. Future work will attempt to gather and analyze data from those sites.

\section{About the Authors}

Hillary Woodworth McNerney graduated with a B.A. in Mass Communication (2006) and a M.A. in Strategic Communication (2019) from the University of Arkansas at Little Rock and is currently pursuing a Ph.D at Regent University in the School of Communication and the Arts. She has previously worked as a journalist at two of the largest newspapers in the state of Arkansas and now works as a program developer for a nonprofit. Her current focus in communication research applies a critical examination of social, political and cultural factors to group communication behaviors, particularly when online group behaviors affect real-world phenomena. Contact her at hillmcn@mail.regent.edu. ORCiD\# 0000-0001-7048-394X

Billy Spann is a Ph.D. Candidate at the University of Arkansas at Little Rock in the Department of Computer and Information Science. He joined the Collaboratorium for Social Media and Online Behavioral Studies (COSMOS) in 2018 where he studies how individuals and groups use social media, blogs, and other digital platforms to coordinate online collective action such as deviant cyber flash mobs, protests, and social movements. His research interests include computational social science, modeling connective action in online social networks, and social-cyber forensics. Contact him at bxspann@ualr.edu. ORCiD\# 0000-0003-1977-8659

Esther L. Mead is a postdoctoral fellow at COSMOS, where she has been a member since 2016. She holds Bachelor's degrees in Psychology, Finance, and Marketing and Master's degrees in Business Administration and Business Information Systems. She has previously worked as a marketing consultant and taught marketing research and consumer behavior at San Diego State University. She earned her doctoral degree in 
2020. Her research interests revolve around machine learning and computational social science. Contact her at elmead@ualr.edu. ORCiD\# 0000-0002-3180-2239

Joseph Kready is pursuing a Master's degree in Computer Science at the University of Texas at Austin where he uses his natural language processing and AI skills to assist in several COSMOS research projects. Joseph also runs the Artificial Intelligence in Arkansas group, which focuses on building the AI community in Arkansas. Contact him at jkready_@ualr.edu. ORCiD\# 0000-0001-6777-3444

Thomas Marcoux received a Master's degree in Computer Science from University of Arkansas at Little Rock in 2015 and is currently a doctoral student, where he focuses on researching and developing technologies to provide insights into online behaviors and decision making through social media analysis. His research interests include consumer behaviors and online mis/dis-information. Contact him at txmarcoux@ualr.edu. ORCiD\# 0000-0001-8443-6618

Nitin Agarwal is the Jerry L. Maulden-Entergy Chair and Distinguished Professor of Information Science with University of Arkansas, Little Rock, USA. He is the Founding Director of the Collaboratorium for Social Media and Online Behavioral Studies (COSMOS) Research Center (https://cosmos.ualr.edu). His research interests include social computing, behavior modeling, social-cybersecurity, health informatics, data mining, AI/ML, and privacy. Contact him at nxagarwal@ualr.edu. ORCiD\# 0000-00025612-4753

\section{Acknowledgments}

This research is funded in part by the U.S. National Science Foundation (OIA-1946391, OIA-1920920, IIS-1636933, ACI-1429160, and IIS-1110868), U.S. Office of Naval Research (N00014-10-1-0091, N00014-14-1-0489, N00014-15-P-1187, N00014-16-12016, N00014-16-1-2412, N00014-17-1-2675, N00014-17-1-2605, N68335-19-C-0359, N00014-19-1-2336, N68335-20-C-0540, N00014-21-1-2121, N00014-21-1-2765, N00014-21-1-2765), U.S. Air Force Research Lab, U.S. Army Research Office (W911NF20-1-0262, W911NF-16-1-0189), U.S. Defense Advanced Research Projects Agency (W31P4Q-17-C-0059), Arkansas Research Alliance, the Jerry L. Maulden/Entergy Endowment at the University of Arkansas at Little Rock, and the Australian Department of Defense Strategic Policy Grants Program (SPGP) (award number: 2020106-094). Any opinions, findings, and conclusions or recommendations expressed in this material are those of the authors and do not necessarily reflect the views of the funding organizations. The researchers gratefully acknowledge the support. 


\section{References}

Abril, D., 2021. Trump supporters flock to MeWe, Gab, and Rumble after Parler goes offline. [online] Fortune. Available at: <https://fortune.com/2021/01/11/mewe-gabrumble-growth-parler-trump-bans-social-media-violence/> [Accessed 18 July 2021].

Agarwal, N., Al-khateeb, S., Galeano, R., and Goolsby, R., 2017. Examining the Use of Botnets and their Evolution in Propaganda Dissemination. Journal of NATO Defense Strategic Communications, 2, pp. 87-112.

Aliapoulios, M., Bevensee, E., Blackburn, J., Bradlyn, B., Cristofaro, E., Stringhini, G. and Zannettou, S., 2021. A Large Open Dataset from the Parler Social Network. [online] Zenodo. Available at: <https://zenodo.org/record/4442460\#.YSF1bN9Ol0j> [Accessed 21 August 2021].

Bastian, M. and Heymann, S., 2009. Gephi: an open-source software for exploring and manipulating networks. [online] Gephi.org. Available at: <https://gephi.org/publications/gephi-bastian-feb09.pdf> [Accessed 21 August 2021].

Blondel, V., Guillaume, J., Lambiotte, R. and Lefebvre, E., 2008. Fast unfolding of communities in large networks. Journal of Statistical Mechanics: Theory and Experiment, 2008(10), pp. 1-12.

Bonilla, Y. and Rosa, J., 2015. \#Ferguson: Digital protest, hashtag ethnography, and the racial politics of social media in the United States. American Ethnologist, 42(1), pp.417.

Chan, M., 2010. The impact of email on collective action: a field application of the SIDE model. New Media \& Society, 12(8), pp.1313-1330.

Daly, K. and Fischer, S., 2021. The online far right is moving underground. [online] Axios. Available at: <https://www.axios.com/the-online-far-right-is-moving-undergrounde429d45d-1b30-46e0-82a3-6e240bf44fef.html> [Accessed 17 July 2021].

Department of Homeland Security, 2020, October. Homeland Threat Assessment.

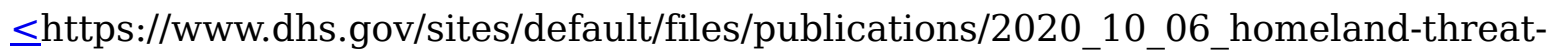
assessment.pdf $>$ [Accessed 17 July 2021].

Der Spiegel., 2019. Far-Right Terrorism in Germany: Shooting Exposes Lapses in Security Apparatus. [online] Available at: <https://www.spiegel.de/international/germany/far-right-terrorism-in-germany- 
shooting-exposes-lapses-in-security-apparatus-a-1291075.html> [Accessed 10 August 2021].

Developer.Twitter.com. 2021. Twitter API for Academic Research Product Track. [online] Available at: <https://developer.Twitter.com/en/products/Twitter-api/academicresearch> [Accessed 31 July 2021].

FBI Phoenix Field Office, 2019. (U//LES). Anti-government, identity based, and fringe political conspiracy theories very likely to motivate some domestic extremists to commit criminal, sometimes violent activity." $\leq$ https://publicintelligence.net/fbiconsiracy-theory-domestic-extremism/> [Accessed 3 August 2021].

Forscher, P. and Kteily, N., 2019. A Psychological Profile of the Alt-Right. Perspectives on Psychological Science, 15(1), pp.90-116.

GitHub. 2021. Detoxity: Toxic Comment Classification with Pytorch Lightning and Transformers. [online] Available at: <https://github.com/unitaryai/detoxify> [Accessed 31 July 2021].

Grover, T., and Mark, G. (2019, July). Detecting Potential Warning Behaviors of Ideological Radicalization in an Alt-Right Subreddit. In Proceedings of the International AAAI Conference on Web and Social Media 13(1), pp. 193-204.

Kready, J., Nihal Hussain, M. and Agarwal, N., 2020. YouTube Data Collection Using Parallel Processing. In: IEEE Workshop on Parallel and Distributed Processing for Computational Social Systems. New Orleans: ParSocial.

Lux, J. and Jordan, J. D., 2019. Alt-Right 'cultural purity', ideology and mainstream social policy discourse: Towards a political anthropology of 'mainstremeist' ideology. In Rees J., Needham C., \& Heins E. (Eds.), Social Policy Review 31: Analysis and Debate in Social Policy, (pp. 151-176). Bristol: Bristol University Press. doi:10.2307/j.ctvkwnq5n.13

Marcoux, T., Galeano, K., Galeano, R., DiCicco, K., Al Rubaye, H., Mead, E., Agarwal, N. and Galeano, A., 2021. A public online resource to track COVID-19 misinfodemic. Social Network Analysis and Mining, 11(1).

Maxwell, T., 2020. Nitter is a new front-end for Twitter that helps hide you from advertisers. [online] Input. Available at: < https://www.inputmag.com/tech/nitter-is-anew-front-end-for-Twitter-that-helps-hide-you-from-advertisers $>$ [Accessed 22 August 2021]. 
Mirrlees, T., 2018. The Alt-right's Discourse on "Cultural Marxism": A Political Instrument of Intersectional Hate. [online] Journals.msvu.ca. Available at: <https://journals.msvu.ca/index.php/atlantis/article/view/5403> [Accessed 18 July 2021].

Obadimu, A., Khaund, T., Mead, E., Marcoux, T. and Agarwal, N., 2021. Developing a socio-computational approach to examine toxicity propagation and regulation in COVID-19 discourse on YouTube. Information Processing \& Management, 58(5), p.102660.

Odağ, Ö., Leiser, A. and Boehnke, K., 2019. Reviewing the Role of the Internet in Radicalization Processes. Journal of Deradicalization, [online] 0(21), pp.261-300. Available at: <https://journals.sfu.ca/jd/index.php/jd/article/view/289> [Accessed 16 July 2021].

Pape, R. A., 2021. Understanding American Domestic Terrorism. Mobilization Potential and Risk Factors of a New Threat Trajectory. UChicago CPOST.

<https://d3qi0qp55mx5f5.cloudfront.net/cpost/i/docs/americas_insurrectionists_online_ 2021_04_06.pdf?mtime=1617807009> [Assessed 16 July 2021].

Postmes, T. and Brunsting, S., 2002. Collective Action in the Age of the Internet. Social Science Computer Review, 20(3), pp.290-301.

Postmes, T., Spears, R. and Lea, M., 1998. Breaching or Building Social Boundaries? Communication Research, 25(6), pp.689-715.

Shahin, S. and Ng, Y., 2020. White Twitter: Tracing the Evolution of the alt-right in Retweets, 2009-2016. Proceedings of the 53rd Hawaii International Conference on System Sciences, [online] Available at: <http://hdl.handle.net/10125/64038> [Accessed 19 August 2021].

Spann, B. and Agarwal, N., 2021. A socio-technical framework for measuring connective action in online social networks. 31st European Conference on Operational Research, session: Web Content and Behaviour, stream: Modelling and Simulation of Social-Behavioural Phenomena in Creative Societies. Athens.

Spears, R. and Postmes, T., 2015. Group Identity, Social Influence, and Collective Action Online. The Handbook of the Psychology of Communication Technology, pp.2346. 
Stanton, Z., 2021. The Problem Isn't Just One Insurrection. It's Mass Radicalization.. [online] POLITICO. Available at: <https://www.politico.com/news/magazine/2021/02/11/mass-radicalization-trumpinsurrection-468746> [Accessed 17 July 2021].

Tajfel, H., and Turner, J. C., 1986. The social identity theory of intergroup behavior. In S. Worchel \& W. G. Austin (Eds.), Psychology of Intergroup Relations (pp. 7-24). Chicago: Nelson Hall. <https://student.cc.uoc.gr/uploadFiles/\%CE\%92310/Tajfel\%20\&\%20Turner\%2086_SIT_ xs.pdf $>$ [Accessed 7 August 2021].

Walther, S. and McCoy, A., 2021. US Extremism on Telegram. Perspectives on Terrorism, [online] 15(2), pp.100-124. Available at: <https://www.jstor.org/stable/27007298> [Accessed 7 August 2021].

\section{Footnotes}

1. https://botometer.osome.iu.edu/ Botscore checker $ヒ$ 